\title{
THREE NEW SOUTH AMERICAN MAILED CATFISHES OF THE GENERA RINELORICARIA AND LORICARIICHTHYS (PISCES, SILURIFORMES, LORICARIIDAE)
}

\author{
by
}

\author{
I. J. H. ISBRƯCKER \& H. NIJSSEN \\ Institute of Taxonomic Zoology, University of Amsterdam, The Netherlands
}

\begin{abstract}
Three new species belonging to two different genera of South American mailed catfishes of the subfamily Loricariinae are described and figured. A discussion of and comparative notes on related species are added.

Rineloricaria formosa $\mathrm{n}$. sp. is described from the Río Inírida/Río Orinoco drainage in Colombia, from the Río Atabapo (Río Orinoco drainage) in Venezuela, and from the Rio Tiquié and Rio Uaupés (Rio Amazonas drainage) in Brazil. It is compared with Rineloricaria morrowi Fowler, 1940, with Rineloricaria melini (Schindler, 1959), and with Rineloricaria fallax (Steindachner, 1915). The lectotype of the latter species is herein designated, and a discussion on the misidentification of $R$. fallax with Loricariicbthys brunneus (Hancock, 1828) is added.

Rineloricaria basemani $\mathrm{n}$. sp. is based on three specimens, two of which are paralectotypes of Rineloricaria fallax from Maguarý. The specimens were collected from streams around Belém, Est. Pará, Brazil. This new species seems more closely related to Rineloricaria stewarti (C. H. Eigenmann, 1909) than to $R$. fallax.

Loricariicbthys platymetopon n. sp. is based upon several specimens found in the Río Paraná/Rio Paraguay system and in the Rio Uruguay, and upon one specimen collected from near the Rio Solimões. This species has hitherto erroneously been recorded as Loricaria maculata by Valenciennes (1840, 1847) and by Boulenger (1896), as Loricariichthys maculatus by Lüling (1975), and as Loricaria typus (in subgenus Loricariichthys) by Regan (1904) and by A. de Miranda Ribeiro (1918). It seems likely that previous records of these nominal species (particularly from the Paraná/Paraguay basin) actually concern, at least in part, L. platymetopon. This is confirmed for two specimens from the Rio Uruguay. L. platymetopon is compared with Loricariichthys maculatus (Bloch, 1794) of which Parabemiodon typus Bleeker, 1862, provisionally is considered a junior synonym - and with Loricariichthys ucayalensis Regan, 1913, the lectotype of which is herein designated from the two syntypes.
\end{abstract}

\section{ABBREVIATIONS USED}

ANSP

Academy of Natural Sciences of Philadelphia, Philadelphia, $\mathrm{Pa}$.

BMNH British Museum (Natural History), London.

CM Carnegie Museum (specimens in FMNH).

FMNH Field Museum of Natural History, Chicago, Ill.

IRScNB Institut Royal des Sciences Naturelles de Belgique, Brussels.
MACN Museo Argentino de Ciencias Naturales "Bernardino Rivadavia", Buenos Aires.

MCZ Museum of Comparative Zoology, Cambridge, Mass.

MNHN Muséum National d'Histoire Naturelle, Paris.

MZUSP Museu de Zoologia da Universidade de São Paulo, São Paulo.

NIU Northern Illinois University, DeKalb, Ill. (specimens in FMNH).

NMW Naturhistorisches Museum, Vienna.

NRS Naturhistoriska Riksmuseet, Stockholm.

RMNH Rijksmuseum van Natuurlijke Historie, Leiden.

USNM National Museum of Natural History, formerly United States National Museum, Washington D.C.

ZFMK Zoologisches Forschungsinstitut und Museum "Alexander Koenig", Bonn.

ZMA Instituut voor Taxonomische Zoölogie (Zoölogisch Museum), Amsterdam.

ZMB Museum für Naturkunde der Humboldt-Universität, Berlin.

ZSM Zoologische Sammlung des Bayerischen Staates, Munich.

hl head length.

si standard length.

\section{INTRODUCTION AND ACKNOWLEDGE- MENTS}

The present publication forms part of a series of studies of the taxa within the subfamily Loricariinae. The methods employed to prepare the descriptions are the same as in our 1978 publication, except for the term "odontode", which substitutes the term "dermal denticle" as previously used (see Ørvig, 1977).

Several colleagues have been helpful in sending specimens in their care on loan, as an exchange, or as a gift. We would like to express our great appreciation for this cooperation to the following persons: Mrs. J. Abel (MNHN), Mr. E. Âhlander (NRS), Mrs. Dr. M. L. Bauchot (MNHN), Dr. M. Boeseman (RMNH), Dr. J. E. Böhlke (ANSP), Dr. H. A. Britski (MZUSP), Dr. H. P. Castello (MACN), Mrs. Ph. Choanier (MNHN), Dr. W. L. Fink (MCZ), Dr. E. J. 
Fittkau (ZSM), Mr. G. S. Glodek (FMNH), Dr. J. P. Gosse (IRScNB), Mrs. T. A. Greenfield (NIU/FMNH), Dr. P. H. Greenwood (BMNH), Mr. G. J. Howes (BMNH), Dr. R. K. Johnson (FMNH), Mrs. S. Karnella (USNM), Mrs. Dr. C. Karrer (ZMB), Dr. P. Kähsbauer (NMW), Dr. E. A. Lachner (USNM), Dr. K. H. Lüling (ZFMK), Mr. F. Scharl (ZSM), Dr. F. Terofal (ZSM), Mr. E. Walschaerts and Mr. L. Walschaerts (IRScNB), and Dr. P. J. P. Whitehead (BMNH).

Mr. L. A. van der Laan (ZMA) made the photographic illustrations and Mr. J. Zaagman (ZMA) made the drawings for the present publication.

We are much obliged to Mr. G. J. Howes (BMNH) who kindly commented upon the typescript of this paper.

\section{FIN RAY COUNTS}

Except for a few aberrant individuals, all the specimens recorded in this paper have the usual fin ray counts for species of Rineloricaria and Loricariichtbys, which they share with species of the genera Loricaria, Ricola, Pseudobemiodon, Rhadinoloricaria, Planiloricaria, Spatuloricaria, Pseudoloricaria, Hemiodontichthys, and Reganella: dorsal fin $I, 6, i$; anal fin $I, 4, i$; pectoral fin $I, 6$; pelvic fin I,5; and caudal fin I,10,I.

The largest paratype of Rineloricaria formosa (FMNH 83714, sl $151.1 \mathrm{~mm}$ ) has the caudal fin with I,9,I rays. Three specimens of Rineloricaria fallax were found with deviating fin ray counts: NMW 44868 ("sl" $138.5 \mathrm{~mm}$ ) has I,8 or 9,I caudal fin rays; ZMA 114.349 (sl $104.5 \mathrm{~mm}$ ) has one of the pelvic fins with $I, 4$ rays, last two rays fused to one; and ZMA 110.662 (nontype, Demerara River, sl $109.4 \mathrm{~mm}$ ) has the caudal fin with I,9,I rays. Finally, the largest paratype of Loricariichthys platymetopon in BMNH 1928.1.6: $1.5(\mathrm{sl} 86.1 \mathrm{~mm})$ has one of the pectoral fins with I,5 rays.

\section{Rineloricaria formosa $n$. sp.}

(Figs. 1-3, 6; tables Ia, IIIa-e)

\section{Material examined. -}

Holotype: FMNH 83713, sl $126.8 \mathrm{~mm}$, Colombia, lagoon about $1 \mathrm{~km}$ upriver from Puerto Inírida, Río Inírida/ Río Orinoco drainage, coll. Thomerson, Hicks, Baskin, Rofen \& Blanco, 28-III-1974.

Paratypes: FMNH 83714 (twenty-two), ZMA 114.922 (five), sl 65.5 to $151.5 \mathrm{~mm}$, same data as holotype; FMNH 83715 (eight), ZMA 114.923 (two), sl 55.8 to $83.8 \mathrm{~mm}$, Colombia, sandbar in Río Inírida, "about 45 minutes upstream from Puerto Inírida", same collectors as for holotype, 29III-1974.

ZSM 25281 (two), ZMA 115.182 (one), sl 79.6 to 127.5 $\mathrm{mm}$, Venezuela, Prov. Amazonas, Río Atabapo near San Fer- nando de Atabapo, $04^{\circ} 03^{\prime} \mathrm{N} 67^{\circ} 45^{\prime} \mathrm{W}$, along Colombian/ Venezuelan border, coll. H. J. Köpke \& M. Jeschke, 5-II-1973. IRScNB 608 (five), ZMA 115.196 (one), sl 70.3 to $140 \mathrm{~mm}$, Brazil, Est. Amazonas, Rio Amazonas drainage, Igarapé Acaraposo, tributary at the right bank of Rio Tiquié, coll. J. P. Gosse, 7-XII-1967; IRScNB 609 (twelve), ZMA 115.197 (one), sl 54.7 to $74.3 \mathrm{~mm}$, Brazil, Est. Amazonas, Rio Amazonas drainage, tributary at the right bank of Rio Uaupés at Trovao, about $20 \mathrm{~km}$ upstream from its mouth, coll. J. P. Gosse, 9-XII-1967.

\section{Description. -}

Morphometric and meristic data of the Colombian specimens are presented in tables Ia and IIIa-e, and are not repeated here.

The paratypes from Venezuela and Brazil were received after the completion of the tables, and we may present their morphometric and meristic variation here. Data of the specimens from Venezuela (ZSM 25281, ZMA 115.182, sl $79.6,113.7$, and $127.5 \mathrm{~mm}$, respectively) are given first, followed in parentheses by those of three specimens from Brazil (IRScNB 608, ZMA 115.196, sl 116.8, 136.5, and 140 $\mathrm{mm}$, respectively): sl 79.6 to 127.5 (116.8 to 140$) \mathrm{mm}$, axial length 88 to 139.2 (127.3 of the smallest specimen) $\mathrm{mm}$, total length of the two smaller specimens 109.4 and 143.7 (135.2 and 157.6, of the smallest and of the largest specimen, respectively) $\mathrm{mm}$, hl 5.5 to 5.7 ( 5.4 to 5.6 ), predorsal length 3.8 to 3.9 (3.6 to 3.8 ), postdorsal length 1.5 (1.5 to 1.6 ), postanal length 1.6 to $1.7(1.7)$, dorsal spine length 4.5 to 4.8 (4.6 to 4.8 ), first dorsal ray 4.5 to 5.0 ( 4.8 to 5.0 ), anal spine length 5.5 to 6.0 ( 5.6 to 6.0 ), pectoral spine length of two larger specimens 5.5 and 5.8 ( 5.9 to 6.0 ), pelvic spine length 6.3 to 6.9 (5.7 to 6.0 ), upper caudal spine of the two smaller specimens 2.7 and 3.8 (of the smallest and the largest specimen 6.3 and 9.9 , respectively), lower caudal spine of the smallest and the largest specimen 7.7 and 8.0, respectively (of the smallest and the largest specimen 8.1 and 8.0, respectively).

Snout length 2.2 (2.1 to 2.2 ), lower lip 6.7 to 8.6 (7.2 to 9.0 ), thoracic length 1.4 to 1.5 ( 1.4 to 1.5$)$, abdominal length 1.3 to 1.4 (1.3 to 1.4 ), maximum orbital diameter 4.0 to 4.2 (4.1), interorbital width 5.6 in the juvenile and 6.0 to 6.5 in the two larger specimens ( 5.7 in the two smaller specimens, 6.1 in the largest specimen), cleithral width 1.3 (1.3), supra-cleithral width 1.8 to 1.9 (1.9 to 2.0 ), head width 1.3 (1.3 to 1.4 ), head depth 2.9 to 3.0 (2.5 to 2.7$)$, body depth at dorsal 2.2 to 2.5 (2.0 to 2.1 ), body width at dorsal 1.5 to 1.6 (1.4 to 1.5$)$, body width at anal 1.7 to 1.8 (1.6 to 1.7), depth caudal peduncle 16.7 to 18.3 (14.0 to 15.7 ), width caudal peduncle 6.9 to 8.5 (6.6 to 7.0$)$, rictal barbel 3.0 to 3.3 ( 3.1 to 3.5 ).

Lateral scutes $32-33 / 32-33(32 / 32)$, coalescing scutes $15 / 15$ (14-15/14-15), thoracic scutes 6-8/5-7 (7-8/7), teeth upper jaws 7-9/7-8 (7-8/7-8), teeth lower jaws 8-9/8-11 (5-8/8-9).

Abdomen completely covered with scutes (also fully developed in the smallest paratype) in three complexes, as follows:

(1) A posterior complex, consisting of a well- 


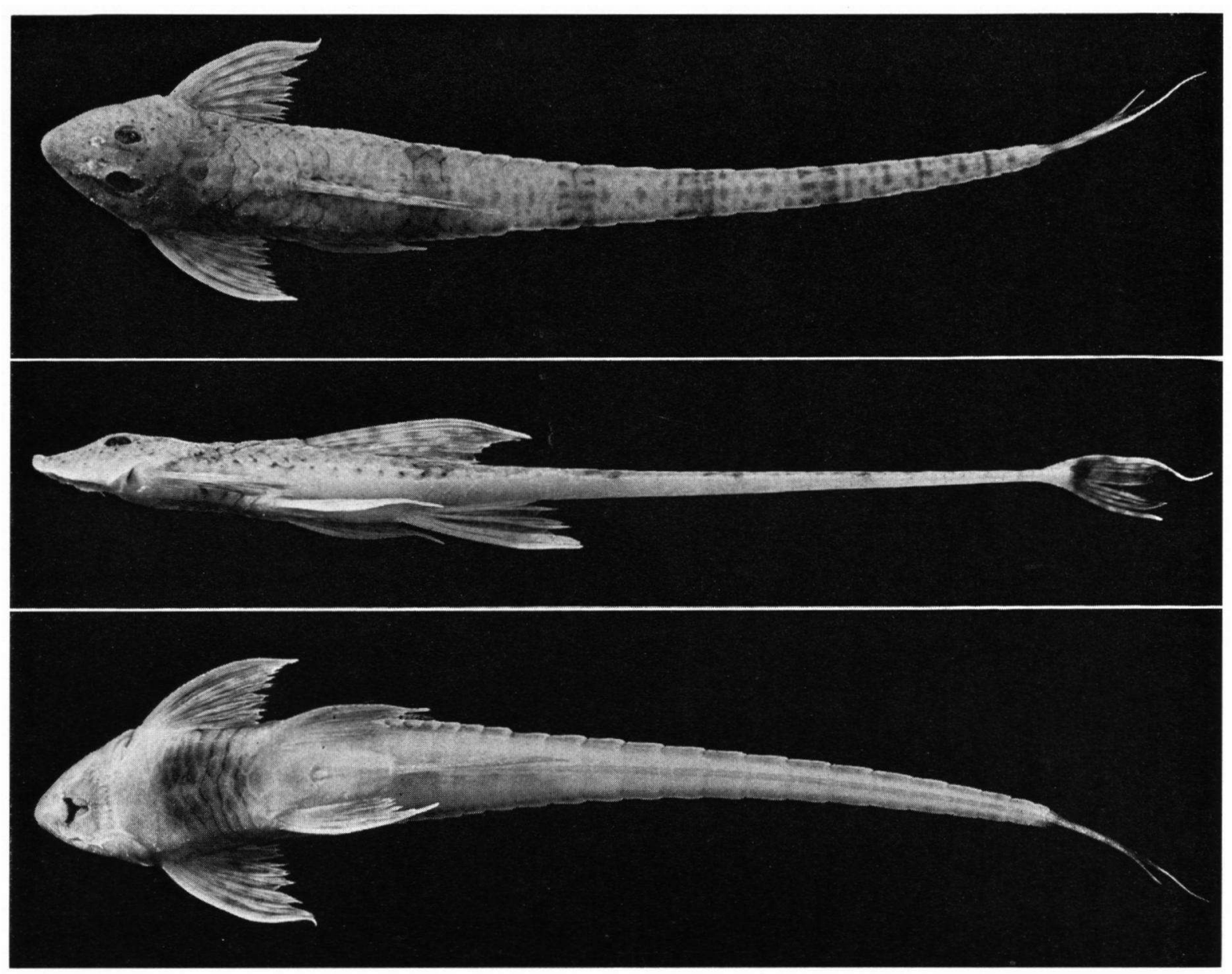

Fig. 1. Rineloricaria formosa n. sp., holotype in dorsal, lateral, and ventral view.

developed preanal plate, preceded by three comparatively large polygonal scutes. Anterior to these scutes are five to six smaller polygonal scutes in a curved transverse series, reaching the posterior thoracic scute. All these elements may together form an inflexible plate, although in some of the specimens the scutes in transverse series allow some movement. This posterior complex is preceded by: (2) A flexible median complex, consisting of small polygonal scutes reaching the ventral edges of the thoracic scutes. There are three median series of four to six scutes in longitudinal rows.

(3) An anterior inflexible complex of still smaller, polygonal scutelets in front of the median complex. This immovable plate is more or less rounded anteriorly and reaches to the height of a ventral extension of the dermal ossification of the head margin, at its broadest part.
The head has ventrally an almost uninterrupted marginal dermal ossification with a more or less triangular extension anterior to the branchiostegal membrane. The tip of the snout has a short, narrow naked horizontal area. All dermal ossifications including scutes, fin spines, and rays - are covered with minute, acute dermal denticles, the odontodes.

Ridges covered with somewhat more prominent odontodes are - as usual throughout the members of the genus Rineloricaria - present in two longitudinal rows along the coalescing and parallel lateral body scutes. In addition, a longitudinal series of very low ridges is visible on six scutes situated between the lateral and the dorsal scutes, the first of which is at the height of the origin of the dorsal fin spine. Two quite prominent, posteriorly strongly diverging ridges are present on the supraoccipital process, and a pair of ridges is 


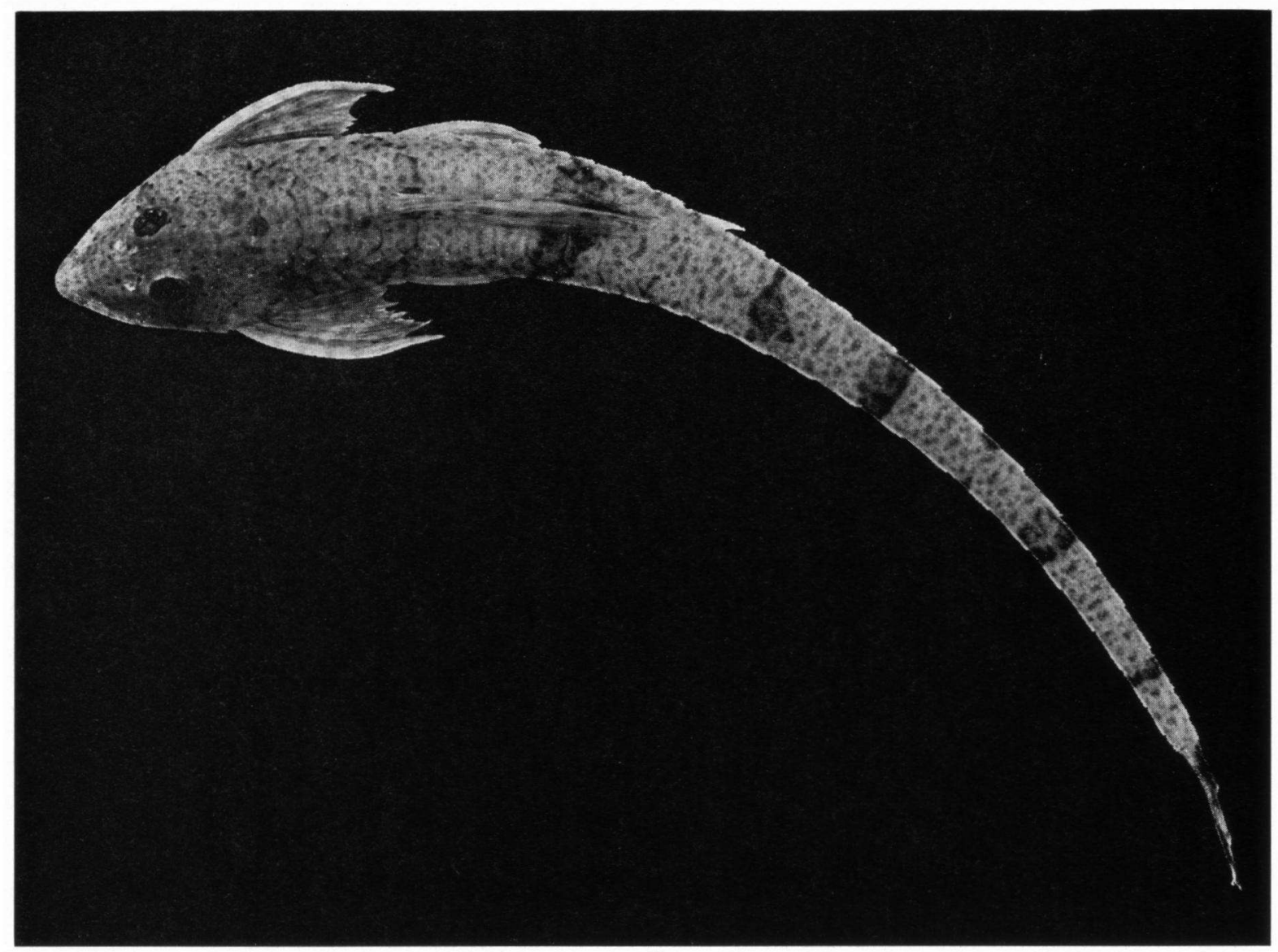

Fig. 2. Rineloricaria formosa n. sp., paratype (FMNH 83715, sI $83.3 \mathrm{~mm}$ ) in dorsal view, showing the more prominent colour pattern of a young specimen.

present on the predorsal scutes between the supraoccipital process and the large median predorsal plate. Dorsal snout margin and supraorbital edges slightly raised; anterior region of predorsal scutes and posterior region of supraoccipital process somewhat depressed. Tip of supraoccipital process round.

A conspicuous posterior orbital notch is present.

A pectoral pore is not found. There are simple pores of the sensory canal system on the dorsum of head and snout, and bifurcated pores between the ridges along the coalescing and parallel lateral body scutes and in a naked area dorsal to the first two coalescing scutes.

The shape and structure of the lips are the same as in the related species of Rineloricaria: Upper lip very narrow, naked dorsally, the edge with short, rounded papillae. Lower lip ventrally with numer- ous distinct, isolated papillae, largest and most numerous towards buccal cavity; surface between these larger papillae covered with minute papillae. Lower lip slightly to distinctly notched medially. A short rictal barbel is present between upper and lower lips, covered with papillae. No fleshy flaps or prominent papillae are found in the buccal cavity.

Teeth in upper jaws somewhat smaller than teeth in lower jaws. The shape of the teeth is the same in both upper and lower jaws, with a smaller, acute outer lobe and a larger inner lobe. The inner lobe may have an acute or slightly roundish tip: mostly these are the larger teeth near the medial side of the jaw; more usually the inner tip has a rather narrow base, broadening anteriorly, with a frontally oblique edge (fig. 3 ). 
Colour in alcohol (figs. 1-2). - Ground colour of ossified parts yellowish tan and of naked parts whitish.

Dorsum of body posterior to the dorsal fin base with five to six regularly distributed transverse brown stripes, the anterior stripe is the broadest, the three stripes posterior to it gradually narrower, whereas the two (or one) posterior to these may be rather obsolete. Dorsum of body and head from tip of snout to base of caudal fin with numerous minute and small brown spots of irregular shape, mostly roundish. These spots are visible as darker brown markings on the transverse stripes. The spots beyond the first transverse stripe tend to be larger than those in front of this stripe. Base of second dorsal fin ray with a rather dark brown, small roundish spot. Even brown pigmentation is visible between the ridges on the supraoccipital process. This dark pigmentation is often surrounded by a narrow unpigmented ring (fig. 2), particularly in specimens smaller than $125 \mathrm{~mm}$ (besides the specimens represented in table III, the largest topotype has a sl of only $88.3 \mathrm{~mm}$ ). Some specimens show a faint spot in front of the dorsal fin spine, the same as the characteristic predorsal spot of Rineloricaria fallax.

Pores on dorsum of snout and head black.

Dorsal fin spine and rays, and dorsum of pectoral fin with faint, irregular greyish brown small dots. Tips of first two branched dorsal fin rays usually with diffuse tan pigmentation. The anal fin spine and the outer half of the rays may show some small brownish spots. Pelvic fin spine with brownish pigmentation, sometimes forming small spots.

Caudal fin base with dark brown pigmentation on and just about the triangular scutelet, and on the small dorsal and ventral caudal scutelets. Caudal fin membrane dark brown pigmented. The rays are alternately spotted with brown and tan, forming either a chess-board pattern, or irregular vertical lines. Upper caudal fin spine usually with brown and yellowish spots.

The ten paratypes in FMNH 83715 and in ZMA 114.923 are generally darker than the 28 other Colombian specimens. They tend to have an additional, rather faint stripe on the dorsum of the body (not unlike the, much stronger developed, stripe present in the holotype of Rineloricaria morrowi, see discussion below), commencing at the spot at the base of the second dorsal fin ray, obliquely running forward to the dorsal ridge along the coalescing scutes.

Etymology. - The specific name formosa (Latin) means beautiful and alludes to the whole fish.

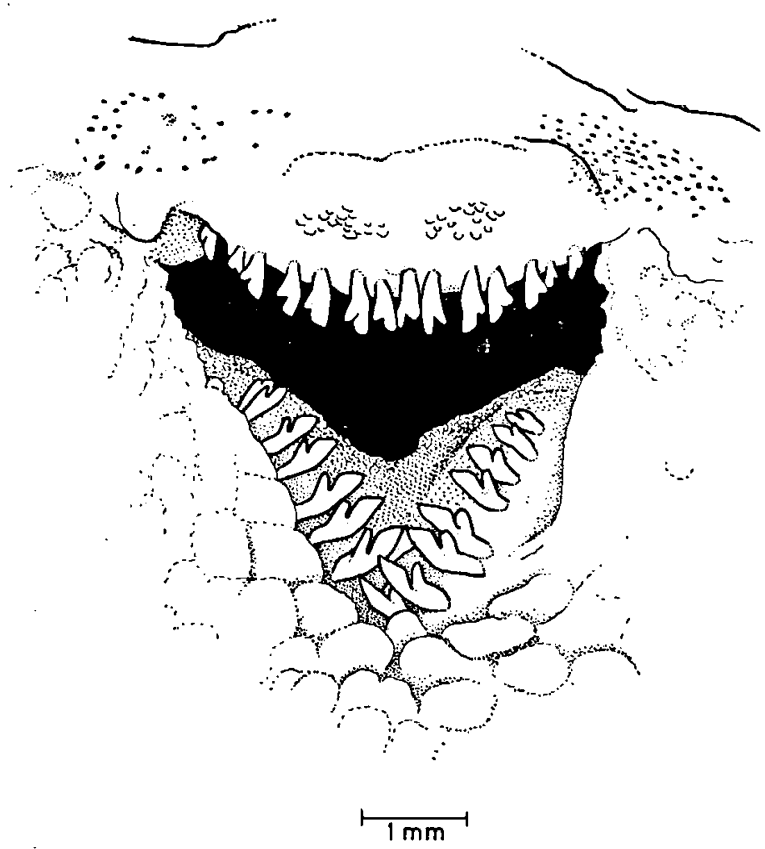

Fig. 3. Rineloricaria formosa n. sp., dentition of the holotype.

Discussion. - Rineloricaria formosa appears on the one hand most closely related to Rineloricaria morrowi Fowler, 1940 and Rineloricaria melini (Schindler, 1959) on the basis of their very similar morphology, especially that of the rounded snout with slightly raised dorsal margin, and the distribution and development of the odontodes, and on the other hand to Rineloricaria fallax (Steindachner, 1915) because of the presence of a predorsal spot.

Rineloricaria morrowi was originally described from the holotype (and still only known specimen, ANSP 68663), collected at Contamana ( $07^{\circ} 19^{\prime} \mathrm{S}$ $75^{\circ} 04^{\prime} \mathrm{W}$ ), Ucayali River basin, Peru (fig. 6). The morphometric and meristic data of this specimen are given in tables Ib and IIIf, showing the differences with $R$. formosa in relative dimensions of predorsal length, postdorsal length, postanal 


\section{TABLE I}

Measurements in $\mathrm{mm}$ to the nearest tenth of: (a) Rineloricaria formosa n. sp., holotype, (b) Rineloricaria morrowi Fowler, 1940, holotype, (c) Rineloricaria melini (Schindler, 1959), holotype, (d) Rineloricaria fallax (Steindachner, 1915), lectotype, (e) Rineloricaria platyura (Müller \& Troschel, in Schomburgk, 1848), holotype, and (f) Rineloricaria platyura, holotype of Loricaria submarginatus $\mathrm{C} . \mathrm{H}$. Eigenmann, 1909.

\begin{tabular}{|c|c|c|c|c|c|c|}
\hline specimen & a & b & c & d & e & $\mathbf{f}$ \\
\hline standard length & 126.8 & 144.2 & 129.5 & 144.7 & 108.4 & 85.1 \\
\hline axial length & 139.2 & 158.3 & 143.4 & 158.0 & 115.2 & 91.6 \\
\hline total length & 150.4 & - & - & - & $>124.5$ & - \\
\hline head length & 21.8 & 26.8 & 22.8 & 26.4 & 20.2 & 16.3 \\
\hline predorsal length & 31.9 & 41.5 & 34.9 & 41.3 & 32.3 & 24.2 \\
\hline postdorsal length & 84.5 & 89.4 & 83.0 & 92.6 & 67.3 & 54.2 \\
\hline postanal length & 77.2 & 78.8 & 73.3 & 81.1 & 59.8 & 48.5 \\
\hline dorsal spine length & 27.7 & $>26.8$ & \pm 26.5 & $>26.5$ & 27.3 & 20.3 \\
\hline first dorsal ray & 26.6 & $>25.6$ & 25.6 & 27.0 & 23.8 & 18.9 \\
\hline anal spine length & 22.0 & 25.9 & 22.4 & 23.3 & 20.8 & 16.5 \\
\hline pectoral spine length & 21.3 & 24.4 & 21.5 & 23.1 & 18.9 & 14.6 \\
\hline pelvic spine length & 20.0 & 24.9 & 20.3 & 21.5 & 18.9 & 14.7 \\
\hline upper caudal spine & 23.6 & - & - & - & $>17.1$ & - \\
\hline lower caudal spine & 16.3 & 21.6 & - & - & $>16.5$ & - \\
\hline snout length & 9.7 & 13.1 & 10.8 & 12.4 & 10.3 & 8.8 \\
\hline lower lip & 3.1 & - & - & 2.4 & - & 2.2 \\
\hline thoracic length & 16.1 & 19.3 & 17.6 & 21.5 & 16.4 & 10.9 \\
\hline abdominal length & 16.3 & 21.4 & 18.5 & 21.5 & 15.3 & 11.3 \\
\hline max. orbital diameter & 5.2 & 6.6 & 5.5 & 6.2 & 5.0 & 3.2 \\
\hline interorbital width & 3.5 & 4.7 & 4.4 & 5.0 & 5.2 & 4.3 \\
\hline cleithral width & 17.4 & 21.2 & 18.3 & 22.9 & 16.2 & 12.4 \\
\hline supra-cleithral width & 12.1 & 14.9 & 12.1 & 14.9 & 12.0 & 9.1 \\
\hline head width & 17.4 & 20.2 & 16.7 & 22.9 & 15.1 & 11.9 \\
\hline head depth & 7.3 & 10.4 & 9.7 & 14.3 & 9.2 & 6.4 \\
\hline body depth at dorsal & 9.3 & 13.1 & 12.2 & 16.0 & 10.7 & 7.5 \\
\hline body width at dorsal & 14.4 & 18.7 & 17.0 & 22.4 & 15.9 & 10.3 \\
\hline body width at anal & 12.6 & 17.1 & 15.3 & 19.2 & 13.7 & 9.6 \\
\hline depth caudal peduncle & 1.4 & 2.1 & 1.7 & 2.4 & 1.3 & 1.1 \\
\hline width caudal peduncle & 3.1 & 4.4 & 3.5 & 4.6 & 2.8 & 2.0 \\
\hline rictal barbel & 6.7 & - & 7.0 & 6.9 & - & 4.7 \\
\hline
\end{tabular}

length, anal spine length, pelvic spine length, snout length, head depth, and depth and width of caudal peduncle.

Although the holotype of Rineloricaria morrowi has a somewhat faded pigmentation, the differences in colour pattern between this species and $R$. formosa are still evident. In $R$. morrowi there are minute, obscure brownish spots on the dorsum of head and body, less prominent than in $R$. formosa. Posterior to the dorsal fin base five conspicuous, transverse, rather broad, solid dark brown stripes are present; in addition, a sixth transverse stripe (far less conspicuous than the other stripes) is present on the dorsum of the body, running obliquely forward from the first to the third branched dorsal fin ray; at the base of these rays a dark brown spot is visible. The spots on dorsal and anal fins are similar to those in $R$. formosa, whereas the dorsum of the pectoral fin hardly has any spots. The caudal fin has a colour pattern resembling that of $R$. formosa, but has a more plain central portion with small, light and indeterminate spots, the distal ends of the branched rays being provided with dark brown pigment forming a vertical stripe along the margin. The base of the fin, including the caudal scutes, is dark brown just like the anterior third of the upper and lower branched rays. Pelvic fin plain. Ventral part of the body devoid of pigmentation, except for some small dark brown marginal concentrations posterior to the 19th lateral body scute. The tip of the supraoccipital process is somewhat darker than the surrounding predorsal region. We should add that this is not clearly visible: a concentration of dark brown pigment has not been noted by Fowler (1940: 244-246, figs. 39-41). Fowler (loc. cit.) originally used the variant (erroneous) spelling of Rhineloricaria, which he subsequently corrected (Fowler, 1954: 118-119, references).

Rineloricaria melini was originally described as Loricariichthys melini by Schindler (1959: 387 . 389 ). It is known only from the holotype (NRS $10830)$, collected near Manacapuru $\left(03^{\circ} 16^{\prime} \mathrm{S}\right.$ $60^{\circ} 37^{\prime} \mathrm{W}$ ), Rio Solimões (= Rio Amazonas), Est. Amazonas, Brazil (fig. 6). Isbrücker \& Nijssen (1976a: 112) listed this species as Rineloricaria melini. We have examined the holotype, of which the morphometric and meristic data are given in tables Ic and IIIg, showing the differences with $R$. formosa in relative dimensions of predorsal length, postdorsal length, postanal length, snout length, abdominal length, interorbital width, head depth, body depth at dorsal, body width at dorsal and anal, and depth of caudal peduncle. $R$. melini has in longitudinal lateral series one scute less than $R$. formosa.

The colour pattern of Rineloricaria melini resembles that of $R$. formosa. $R$. melini has some transverse dark brown stripes on the dorsum of the body, but these are quite irregular and hard to distinguish from the large, irregular dark brown blotches also present on the dorsum of the body. There is a very narrow, incomplete transverse stripe on the 18th, one stripe on the 19th, one on the 
22nd, and one on the 28th lateral body scute. The spots and blotches on the dorsum of head and body are much more prominent than in both $R$. formosa and $R$. morrowi, those on the head forming at least four transverse, narrow, rather irregularly defined stripes. A conspicuous though small, dark brown spot is present at the base of the first branched dorsal fin ray. Caudal fin damaged, but showing traces of a colour pattern resembling that of $R$. morrowi rather than that of $R$. formosa. Colour pattern of pectoral and dorsal fins as in $R$. formosa, pelvic and anal fins without pigmentation.

The small but often conspicuous dark spot between the ridges on the supraoccipital process in $R$. formosa, $R$. morrowi and $R$. melini - which is almost always surrounded by a narrow unpigmented ring in the freshly preserved specimens of $R$. formosa - is reminiscent of a characteristic pattern found in Rineloricaria fallax (Steindachner, 1915). In this species, there is a conspicuous dark brown spot in front of the dorsal fin. This spot is surrounded by a narrow, unpigmented ring, which is laterally bordered with a dark brown, partly roundish line extending sometimes anteriorly as far as the tip of the supraoccipital process. In few specimens this colour mark is rather faint.

In a subsequent paper we will redescribe and illustrate Rineloricaria fallax. We take the opportunity to discuss here some taxonomic problems concerning this species and Loricariichtbys brunneus (Hancock, 1828), with which Rineloricaria fallax has been often confused.

\section{Rineloricaria fallax (Steindachner, 1915)}

(Fig. 6; tables Id, IIIh)

[Loricaria] brunnea (non Hancock, 1828); C. H. Eigenmann, 1909: 10 \& 11 (in original descriptions of Loricaria stewarti and Loricaria submarginatus; comparison).

Loricariichthys brunneus (non Hancock, 1828); C. H. Eigenmann, 1912: 247-248, pl. XXX fig. 3, pl. XXXI fig. 4 (description; 75 specimens, up to $183 \mathrm{~mm}$; British Guiana: Lama stop-off, Maduni Creek, Botanic Garden, Demerara River below Wismar, Christianburg Canal, Wismar, Kumaka, Demerara, Malali, Gluck Island, Rockstone, Tumatumari, Rupununi; in key on p. 245 , in subgenus Rineloricaria; also recorded on pp. 15, 67, 77, 88 \& 96).

Loricaria brunnea (non Hancock, 1828); C. H. Eigenmann \& Allen, 1942: 203-204 (Guianas and Peru; 2 specimens, up

\section{TABLE II}

Measurements in $\mathrm{mm}$ to the nearest tenth of: (a) Rineloricaria basemani n. sp., holotype, (b) Rineloricaria stewarti (C. $\mathbf{H}$. Eigenmann, 1909), holotype, (c) Loricariicbthys platymetopon n. sp., holotype, (d) Loricariicbtbys maculatus (Bloch, 1794), lectotype, (e) Loricariichthys maculatus, holotype of Parabemiodon typus Bleeker, 1862, and (f) Loricariichthys ucayalensis Regan, 1913, lectotype.

\begin{tabular}{|c|c|c|c|c|c|c|}
\hline specimen & a & b & c & d & $\mathbf{e}$ & $\mathbf{f}$ \\
\hline standard length & 136.9 & 57.3 & 222.3 & 212.5 & 177.0 & 198.0 \\
\hline axial length & 150.5 & 63.3 & 246.1 & - & - & 217.0 \\
\hline total length & 200.9 & - & - & - & - & 230.9 \\
\hline head length & 26.1 & 12.0 & 51.2 & 43.7 & 37.2 & 47.0 \\
\hline predorsal length & 39.5 & 17.2 & 74.2 & 65.4 & 55.4 & 68.6 \\
\hline postdorsal length & 84.8 & 35.5 & 125.5 & 129.1 & 105.8 & 108.5 \\
\hline postanal length & 74.2 & 30.9 & 108.8 & 112.5 & 91.4 & 94.0 \\
\hline dorsal spine length & 31.7 & 11.2 & 49.8 & 43.8 & - & 42.0 \\
\hline first dorsal ray & 31.0 & 10.9 & 48.7 & 42.8 & - & 40.6 \\
\hline anal spine length & 25.5 & 9.6 & 39.5 & 36.1 & - & 37.1 \\
\hline pectoral spine length & 23.6 & 9.4 & 35.7 & 31.6 & - & 32.5 \\
\hline pelvic spine length & 22.3 & 8.7 & 33.4 & 36.0 & \pm 29.6 & 31.7 \\
\hline upper caudal spine & 64.0 & - & - & - & - & $>33.8$ \\
\hline lower caudal spine & 19.2 & 8.5 & - & - & - & 29.3 \\
\hline snout length & 12.4 & 5.6 & 25.0 & 21.9 & 19.3 & 25.6 \\
\hline lower lip & 4.2 & 1.6 & 25.3 & 20.6 & 20.1 & 31.6 \\
\hline thoracic length & 20.1 & 8.8 & 35.2 & 31.6 & 26.6 & 30.6 \\
\hline abdominal length & 21.5 & 7.6 & 32.6 & 28.9 & 25.0 & 27.5 \\
\hline $\max$. orbital diameter & 6.4 & 2.5 & 11.9 & 10.1 & 9.3 & 10.9 \\
\hline interorbital width & 4.7 & 2.7 & 15.5 & 9.8 & 9.1 & 11.4 \\
\hline cleithral width & 21.6 & 8.7 & 41.2 & 32.0 & 28.6 & 36.7 \\
\hline supra-cleithral width & 14.8 & 6.1 & 34.8 & 26.1 & 22.7 & 28.7 \\
\hline head width & 21.6 & 8.7 & 41.2 & 32.0 & 28.6 & 36.8 \\
\hline head depth & 11.5 & 4.3 & 23.3 & 18.3 & 16.2 & 17.9 \\
\hline body depth at dorsal & 14.0 & 4.9 & 28.6 & 22.3 & 20.1 & 19.6 \\
\hline body width at dorsal & 18.1 & 7.6 & 36.0 & 29.2 & 25.4 & 32.1 \\
\hline body width at anal & 16.9 & 5.9 & 30.7 & 26.7 & 23.8 & 25.3 \\
\hline depth caudal peduncle & 2.0 & 1.0 & 6.7 & 3.7 & 3.8 & 4.3 \\
\hline width caudal peduncle & 3.7 & 1.6 & 8.5 & 7.4 & 6.1 & 7.7 \\
\hline rictal barbel & 8.9 & - & 24.7 & 25.4 & - & 24.0 \\
\hline
\end{tabular}

to $109 \mathrm{~mm}$; Peru: Yarinacocha, description; 3 specimens, up to $123 \mathrm{~mm}$; Peru: Rio Pacaya and Iquitos, description; comparative note on "types" taken in British Guiana [ = Loricariichtbys brunneus sensu C. H. Eigenmann, 1912]); Fowler, 1945: 104 (listed; Perú, Yarinacocha, Rio Pacaya, Iquitos; Río Orinoco); Gosline, 1945: 105 (listed; in subgenus Loricariichtbys; Guiana Inglêsa); Van der Stigchel, 1946 \& 1947: 179-180 (description; 2 specimens recorded by C. H. Eigenmann, 1912); Tovar Serpa, 1967: 221 (listed; after Fowler, 1945; also listed on p. 222).

Loricariichthys brunnea (non Hancock, 1828); Schindler, 1959: 389 (in original description of Loricariichtbys melini; comparison).

Loricaria (Loricariicbtbys) brunnea (non Hancock, 1828); Boeseman, 1976: 165-167, pls. V \& VII, table 3 (references to Hancock, 1828, C. H. Eigenmann, 1912, and Van der Stigchel, 1946 \& 1947; 23 specimens, up to $127 \mathrm{~mm}$ sl from Surinam: Corantijn River basin, Winanna Creek, Avanavero Falls, tributaries of Kabalebo River, Morali Creek, right tributary of Kaboeri Creek; southwestern Surinam: Sipaliwini River; southern Surinam or northern Brazil: jungle creek near Apisikè; discussion; in key on p. 156; pls. V \& 
VII with caption Loricaria (Loricariichtbys?) brunnea; recorded as Loricaria brunnea on pp. 166, 167 \& 168; affinity with Rineloricaria suggested on p. 167).

Loricaria (Loricariichthys) fallax Steindachner, 1915: 101. 103, pl. XIII figs. 1.4 (original description; pro parte; new name followed by a question mark; syntype localities: "Rio Rupununi, dem Rio Parimé bei Serra do Banco, aus einem Igarapé bei Capivary, dem Rio branco bei Bem Querer und Conçeicaõ sowie aus einem Flusse bei Maguarý an der Braganzabahn bei Pará"; doubtful synonymy of and comparison with Loricariichthys bsunneus sensu C. $H$. Eigenmann, 1912 and Loricaria or Loricariichtbys stewarti; Steindachner agrees with C. H. Eigenmann's description, but not with his illustration in 1912, pl. XXX fig. 3, of Loricariichtbys brunneus); Schindler, 1959: 389 (in original description of Loricariichtbys melini; discussion).

Loricaria fallax; Steindachner, 1915, pl. XIII (caption of plate); Gosline, 1945: 105 (listed; in subgenus Loricariichthys; Rupununi, Rio Parime, Capivarí, Rio Branco, Pará; date of publication given as 1917).

Loricariicbthys fallax; Fowler, 1954: 102-103, fig. 705 (references; figures after Fleischmann, in Steindachner, 1915; Pará, Rio Branco, Guianas).

Rineloricaria fallax; Isbrücker \& Nijssen, 1976a: 112 (listed).

Type-material examined. -

Rineloricaria fallax (Steindachner, 1915):

Lectotype (by present designation): NMW 44864, sl $144.7 \mathrm{~mm}$, male, Brazil, Est. Roraíma, Igarapé de Carauná (= Sa. Grande, $\left.02^{\circ} 35^{\prime} \mathrm{N} 60^{\circ} 45^{\prime} \mathrm{W}\right)$ near Bôa Vista $\left(02^{\circ} 51^{\prime} \mathrm{N}\right.$ $\left.60^{\circ} 43^{\prime} \mathrm{W}\right)$, altitude $200 \mathrm{~m}$ above sea level, Rio Branco drainage, Rio Amazonas basin, coll. J. D. Haseman, about 4-XII-1912.

Paralectotypes: Except for the specimens from Rupununi River, all paralectotypes conspecific with the lectotype originate from Brazil, Est. Roraíma, upper Rio Branco drainage; all were collected by J. D. Haseman, 1912/1913: ZMA 114.349 (ex NMW 46158, two), sl $104.5 \mathrm{~mm}$, Igarapé de Capivary [locality not traced], probably between Rio Parimé and Rio Surumú, III/IV-1913; NMW 46159 (one), sl 79.1 $\mathrm{mm}$, Rio Branco near Bem Querer [locality not traced, but it is near Caracarai, $\left.01^{\circ} 47^{\prime} \mathrm{N} 61^{\circ} 11^{\prime} \mathrm{W}\right]$, 1912; NMW 44868, posterior caudal peduncle somewhat malformed, "sl" 138.5 $\mathrm{mm}$, male, Rio Branco near Conçeicao, $02^{\circ} 10^{\prime} \mathrm{N} 60^{\circ} 55^{\prime} \mathrm{W}$, XII-1912; NMW 44867 (one), sl 133.2 mm, ZSM 4869 (ex NMW), sl $140.8 \mathrm{~mm}$, male, NMW 45046, sl $157.2 \mathrm{~mm}$, male, Rio Branco near Bôa Vista, $02^{\circ} 51^{\prime} \mathrm{N} 60^{\circ} 43^{\prime} \mathrm{W}, 1912$ / 1913.

NMW 46157 (one), sl $134.1 \mathrm{~mm}$, male, and NMW 44866, sl $80.1 \mathrm{~mm}$, southern Guyana near Brazilian frontier (Rio Branco territory), Rupununi River, II-1913.

Rineloricaria platyura (Müller \& Troschel, in Schomburgk, 1848):

NMW 44869 (paralectotype of Loricaria (Loricariicbtbys) fallax), sl $112 \mathrm{~mm}$, Brazil, Est. Pará, Maguarý [locality not traced] along the Bragança railroad [Bragança, $01^{\circ} 02^{\prime} \mathrm{S}$ $\left.46^{\circ} 46^{\prime} \mathrm{W}\right]$, in forest streams which empty into Rio Guamá [Guamá, $01^{\circ} 35^{\prime} \mathrm{S} 47^{\circ} 29^{\prime} \mathrm{W}$ ], 29-VII-1913 (see table IVc).

We have also examined the holotype of this species (ZMB 3166 , sl $108.4 \mathrm{~mm}$, from Guyana, Rupununi River (fig. 6), coll. R. Schomburgk; see tables Ie and IIIi), as well as the holotype of Loricaria submarginatus C. H. Eigenmann, 1909
(FMNH 53080, ex CM 1570, sl $85.1 \mathrm{~mm}$, Guyana, creek below Potaro Landing, Essequibo River system (fig. 6), coll. E. Shideler; see tables If and IIIj). L. submarginatus was rightly considered a junior synonym of $R$. platyura by C. H. Eigenmann (1912: 248-249, in key on p. 245, as Loricariichthys (Rineloricaria) platyurus, pl. XXX fig. 4, pl. XXXI fig. $3)$. The three specimens agree perfectly in colour pattern. The specimen in NMW 44869 differs from the holotypes mentioned in the following morphometric characters: sl, axial length, hl, predorsal length, postdorsal length, postanal length, dorsal spine length, first dorsal ray, anal spine length, pelvic spine length, maximum orbital diameter, interorbital width, head depth, depth caudal peduncle, and rictal barbel. More specimens are needed to assess the taxonomic significance of these apparently slight differences.

Rineloricaria basemani $\mathrm{n}$. sp.:

NMW 44865 and ZSM 19732 are paralectotypes of Loricaria (Loricariichthys) fallax. See description below.

The references to Rineloricaria fallax may prove to concern a polyspecific group after all specimens mentioned have been re-examined and compared (particularly some of the Peruvian material recorded by C. H. Eigenmann \& Allen, 1942). None of these records, however, seems to include specimens belonging to the genus Loricariichthys, but rather to the genus Rineloricaria sensu lato (including Hemiloricaria Bleeker, 1862). This was already suggested by C. H. Eigenmann (1912: 245, in key), who regarded Rineloricaria a subgenus of Loricariichthys.

Steindachner (1915: 101) included Loricaria stewarti (or Loricariichtbys stewarti, also a Rineloricaria sensu lato) and an illustration of Loricariichthys brunneus sensu C. H. Eigenmann, 1912, as doubtful synonyms of his new species. It is interesting to note that two of the numerous syntypes of Loricaria (Loricariichtbys) fallax, although greatly resembling Rineloricaria stewarti, in fact represent a new species, described below as Rineloricaria basemani, whereas another syntype is closely related to (and perhaps identical with) Rineloricaria platyura (Müller \& Troschel, in Schomburgk, 1848). These three syntypes are indicated to be from Maguarý near Pará, a locality remote from the type-localities of both $R$. platyura and $R$. stewarti (Rupununi River, Essequibo River system, Guyana, and Chipoo Creek, a tributary of the Rio Ireng, upper Rio Branco drainage, Rio Amazonas system, Guyana/Brazil, Est. Roraima, respectively; see fig. 6 ).

Steindachner (1915) unfortunately did not state 
TABLE III

Morphometric and meristic data of: (a) Rineloricaria formosa n. sp., holotype, (b-e) Rineloricaria formosa n. sp., four paratypes in FMNH 83714 and ZMA 114.922, (f) Rineloricaria morrowi Fowler, 1940, holotype, (g) Rineloricaria melini (Schindler, 1959), holotype, (h) Rineloricaria fallax (Steindachner, 1915), lectotype, (i) Rineloricaria platyura (Müller \& Troschel, in Schomburgk, 1848), holotype, (j) Rineloricaria platyura, holotype of Loricaria submarginatus C. H. Eigenmann, 1909, (k) Rineloricaria platyura, NMW 44869, (1) Rineloricaria basemani n. sp., holotype, (m-n) Rineloricaria basemani, the 2 paratypes, and (o) Rineloricaria stewarti (C. H. Eigenmann, 1909), holotype.

\begin{tabular}{|c|c|c|c|c|c|c|c|c|c|c|c|c|c|c|c|}
\hline specimen & $\mathbf{a}$ & $\mathbf{b}$ & c & d & e & $f$ & $\mathbf{g}$ & $\mathbf{h}$ & $\mathbf{i}$ & j & k & 1 & m & $\mathrm{n}$ & ० \\
\hline standard length & 126.8 & 151.5 & 125.8 & 125.4 & 66.8 & 144.2 & 129.5 & 144.7 & 108.4 & 85.1 & 112.0 & 136.9 & 116.4 & 103.2 & 57.3 \\
\hline axial length & 139.2 & 165.9 & 138.5 & 137.6 & - & 158.3 & 143.4 & 158.0 & 115.2 & 91.6 & 120.9 & 150.5 & 128.1 & 113.2 & 63.3 \\
\hline total length & 150.4 & 177.2 & - & - & - & - & - & $->$ & $>124.5$ & - & - & 200.9 & - & 144.7 & - \\
\hline head length & 5.8 & 5.6 & 5.7 & 5.7 & 5.3 & 5.4 & 5.7 & 5.5 & 5.4 & 5.2 & 5.0 & 5.2 & 5.2 & 5.2 & 4.8 \\
\hline predorsal length & 4.0 & 3.8 & 3.9 & 4.0 & 3.8 & 3.5 & 3.7 & 3.5 & 3.4 & 3.5 & 3.3 & 3.5 & 3.4 & 3.5 & 3.3 \\
\hline postdorsal length & 1.5 & 1.5 & 1.5 & 1.5 & 1.5 & 1.6 & 1.6 & 1.6 & 1.6 & 1.6 & 1.7 & 1.6 & 1.6 & 1.6 & 1.6 \\
\hline postanal length & 1.6 & 1.7 & 1.7 & 1.7 & 1.6 & 1.8 & 1.8 & 1.8 & 1.8 & 1.8 & 1.9 & 1.8 & 1.8 & 1.8 & 1.9 \\
\hline dorsal spine length & 4.6 & 5.1 & 4.8 & 4.6 & 4.8 & $<5.4$ & \pm 4.9 & $<5.5$ & 4.0 & 4.2 & 4.5 & 4.3 & 4.4 & 4.4 & 5.1 \\
\hline first dorsal ray & 4.8 & 5.2 & 4.8 & 4.7 & 4.9 & $<5.6$ & 5.1 & 5.4 & 4.6 & 4.5 & 4.7 & 4.4 & 4.6 & 4.5 & 5.3 \\
\hline anal spine length & 5.8 & 6.1 & 5.7 & 5.8 & 6.1 & 5.6 & 5.8 & 6.2 & 5.2 & 5.2 & 5.3 & 5.4 & 5.5 & 5.2 & 6.0 \\
\hline pectoral spine length & 6.0 & 6.1 & 5.9 & 5.8 & 6.7 & 5.9 & 6.0 & 6.3 & 5.7 & 5.8 & 5.8 & 5.8 & 6.0 & 6.0 & 6.1 \\
\hline pelvic spine length & 6.3 & 6.2 & 6.3 & 6.2 & 7.6 & 5.8 & 6.4 & 6.7 & 5.7 & 5.8 & 6.1 & 6.1 & 5.9 & 6.0 & 6.6 \\
\hline upper caudal spine & 5.4 & 5.9 & - & - & - & - & - & - & $<6.3$ & - & - & 2.1 & - & 2.5 & - \\
\hline lower caudal spine & 7.8 & - & 7.4 & 7.8 & 8.2 & 6.7 & - & - & $<6.6$ & - & 6.9 & 7.1 & 6.8 & 6.8 & 6.7 \\
\hline snout length & 2.2 & 2.2 & 2.2 & 2.2 & 2.3 & 2.1 & 2.1 & 2.1 & 2.0 & 1.9 & 1.9 & 2.1 & 2.1 & 2.1 & 2.1 \\
\hline lower lip & 7.0 & 7.3 & 6.9 & 7.6 & 8.4 & - & - & 11.0 & - & 7.4 & 5.8 & 6.2 & 5.0 & 5.7 & 7.5 \\
\hline thoracic length & 1.4 & 1.4 & 1.2 & 1.3 & 1.5 & 1.4 & 1.3 & 1.2 & 1.2 & 1.5 & 1.4 & 1.3 & 1.4 & 1.6 & 1.4 \\
\hline abdominal length & 1.3 & 1.3 & 1.3 & 1.3 & 1.6 & 1.3 & 1.2 & 1.2 & 1.3 & 1.4 & 1.4 & 1.2 & 1.3 & 1.4 & 1.6 \\
\hline max. orbital diameter & 4.2 & 4.2 & 3.7 & 4.0 & 4.2 & 4.1 & 4.2 & 4.3 & 4.0 & 4.2 & 4.4 & 4.1 & 4.2 & 4.2 & 4.8 \\
\hline interorbital width & 6.4 & 5.6 & 5.9 & 5.6 & 5.5 & 5.7 & 5.2 & 5.3 & 3.9 & 3.8 & 4.0 & 5.6 & 5.4 & 5.0 & 4.4 \\
\hline cleithral width & 1.3 & 1.3 & 1.2 & 1.3 & 1.4 & 1.3 & 1.3 & 1.2 & 1.2 & 1.3 & 1.3 & 1.2 & 1.2 & 1.3 & 1.4 \\
\hline supra-cleithral width & 1.8 & 1.9 & 1.8 & 1.8 & 1.9 & 1.8 & 1.9 & 1.8 & 1.7 & 1.8 & 1.8 & 1.8 & 1.7 & 1.8 & 2.0 \\
\hline head width & 1.3 & 1.3 & 1.2 & 1.3 & 1.4 & 1.3 & 1.4 & 1.2 & 1.3 & 1.4 & 1.3 & 1.2 & 1.2 & 1.3 & 1.4 \\
\hline head depth & 3.0 & 2.7 & 3.0 & 2.8 & 2.8 & 2.6 & 2.4 & 1.8 & 2.2 & 2.6 & 2.1 & 2.3 & 2.2 & 2.2 & 2.8 \\
\hline body depth at dorsal & 2.3 & 2.1 & 2.2 & 2.2 & 2.7 & 2.1 & 1.9 & 1.7 & 1.9 & 2.2 & 1.9 & 1.9 & 1.8 & 2.0 & 2.5 \\
\hline body width at dorsal & 1.5 & 1.4 & 1.5 & 1.5 & 1.9 & 1.4 & 1.3 & 1.2 & 1.3 & 1.6 & 1.4 & 1.4 & 1.4 & 1.6 & 1.6 \\
\hline body width at anal & 1.7 & 1.6 & 1.7 & 1.7 & 2.2 & 1.6 & 1.5 & 1.4 & 1.5 & 1.7 & 1.6 & 1.5 & 1.5 & 1.8 & 2.0 \\
\hline depth caudal peduncle & 15.6 & 14.3 & 14.7 & 15.7 & 18.0 & 12.8 & 13.4 & 11.0 & 15.5 & 14.8 & 17.1 & 13.1 & 13.1 & 15.3 & 12.0 \\
\hline width caudal peduncle & 7.0 & 6.6 & 6.5 & 7.1 & 8.4 & 6.1 & 6.5 & 5.7 & 7.2 & 8.2 & 7.9 & 7.1 & 6.7 & 8.0 & 7.5 \\
\hline rictal barbel & 3.3 & 3.2 & 3.1 & 3.3 & 3.3 & - & 3.3 & 3.8 & - & 3.5 & 3.0 & 2.9 & 3.2 & 3.0 & - \\
\hline lateral scutes & $32 / 32$ & $32 / 32$ & $33 / 32$ & $32 / 32$ & $32 / 32$ & $32 / 32$ & $31 / 31$ & $32 / 31$ & $30 / 30$ & $30 / 30$ & $30 / 30$ & $33 / 32$ & $32 / 32$ & $32 / 32$ & $31 / 31$ \\
\hline coalescing scutes & $15 / 15$ & $15 / 15$ & $16 / 16$ & $15 / 14$ & $15 / 15$ & $16 / 16$ & $15 / 15$ & $15 / 15$ & $13 / 13$ & $13 / 14$ & $13 / 13$ & $17 / 15$ & $16 / 16$ & $16 / 16$ & $15 / 15$ \\
\hline thoracic scutes & $9 / 8$ & $6 / 8$ & $8 / 8$ & $8 / 8$ & $6 / 6$ & $7 / 6$ & $7 / 7$ & $8 / 7$ & $8 / 8$ & $7 / 8$ & $8 / 7$ & $9 / 8$ & $8 / 7$ & $10 / 9$ & $7 / 7$ \\
\hline teeth upper jaws & $7 / 8$ & $9 / 9$ & $7 / 8$ & $7 / 8$ & $6 / 5$ & $8 / 9$ & $>4 />3$ & $7 / 8$ & $6 / 9$ & $9 / 9$ & $8 / 8$ & $8 / 9$ & $7 / 6$ & $7 / 7$ & $7 / 7$ \\
\hline teeth lower jaws & $10 / 9$ & $9 / 8$ & $8 / 7$ & $9 / 9$ & $7 / 7$ & $5 / 9$ & $8 / 9$ & $9 / 10$ & $10 / 12$ & $6 / 8$ & $8 / 8$ & $8 / 8$ & $7 / 8$ & $8 / 7$ & $10 / 9$ \\
\hline
\end{tabular}

the number of specimens he had available for his original description, and omitted at least some locality records (e.g. from the lectotype; we suspect that this specimen was with the artist who illustrated it while Steindachner made his description). Dr. P. Kähsbauer kindly put at our disposal an unpublished, typewritten report entitled: "The general account of a fishing expedition to northeastern South America, 1912-1913" by J. D. Haseman (24 pages, of which p. 3 is missing). Haseman collected most of the specimens described by Steindachner (1915), including all his Loricaria fallax. Haseman (op. cit.: 19) wrote: "I planned to leave Serra do Mel early April 14 [1913], but the pack animals could not be found until quite late; however we travelled to the end of Serra do Banco before night. We slept there and started at sunrise of the following day so that we would get out of the hot basin between Serra do Banco and Serra de Panelão before noon. Along the way we caught a queer bearded Loricaria, which was probably a male of $L$. brunneus, in a puddle of a dried up stream flowing to Rio Parime." Unfortunately, we did not succeed in finding this specimen among the rich NMW material during visits to the collections of the Naturhistorisches Museum, Vienna. We have examined the syntypes listed above (and designated the lectotype; second 
specimen in Steindachner's table on pp. 102-103, illustrated in Steindachner, 1915, pl. XIII figs. $1-3$; note partly erroneous sex indications in caption to this plate; locality not mentioned by Steindachner, but no other specimen can represent the one figured; see tables Id and IIIh in the present paper for its morphometric and meristic data); notes additional to the localities published by Steindachner are derived from Haseman's unpublished report and from locality labels.

Rineloricaria fallax is a rather common species. In most museum collections, specimens have been misidentified as Loricaria brunnea or as Loricariichthys brunneus. In the list of paralectotypes above, numbers in parentheses following the register numbers refer to additional specimens of the same sample retained in NMW, which were not examined in detail. The other paralectotypes have been examined together at one time. The specimens from Bôa Vista are considered as paralectotypes. Notwithstanding the fact that Bôa Vista was not included in Steindachner's series of syntype-localities, these specimens were collected by J. D. Haseman along with several others in the Rio Branco drainage. All were initially identified - either by Haseman or by Steindachner - as Loricaria (Loricariicbtbys) brunnea prior to the establishment of Rineloricaria fallax. Parts of the material that evidently represent syntypes were still labeled as $L$. brunnea in the Vienna collection. However, no specimens from “...dem Rio Parimé bei Serra do Banco...," were found with a label accordingly written.

\section{Loricariichthys brunneus (Hancock, 1828)}

Loricaria brunnea Hancock, 1828: 247 (original description, based on holotype only; type-locality: Venezuela, "...the branches and lakes of the Orinoko"; length 10 or 12 inches; comparison with Loricaria cataphracta and Loricaria maculata, both sensu Bloch, 1794; holotype lost); Valenciennes, in Cuvier \& Valenciennes, 1840: 479 (: 353-354 in the Strasbourg edition) (description, after Hancock, 1828; "l'Orénoque"); Kner, 1854: 76 (listed); Günther, 1864: 260 (description, after Hancock, 1828; Orinoco); C. H. Eigenmann \& R. S. Eigenmann, 1889: 37 (listed; in subgenus: "?"); C. H. Eigenmann \& R. S. Eigenmann, 1890: 370 (listed; in key on p. 362, after Hancock, 1828; in subgenus Rineloricaria, but probably placed there inadvertently, cf. the same authors, 1899); C. H. Eigenmann \& R. S. Eigenmann, 1891: 39 (listed; in subgenus Rineloricaria; erroneously recorded from Demerara); Regan, 1904: 270 (listed as an insufficiently described species, “...allied to L. maculata Bl."); C. H. Eigenmann, 1910: 415 (listed; in subgenus Loricaria; erroneously recorded from British Guiana); Schultz, 1944: 322 (footnote; reference to Cuvier \& Valenciennes's and Günther's (1864) record of the species from the Orinoco; discussion).

Loricaria brunea; Bleeker, 1858: 331 (listed; Americ. merid.). L[oricariichtbys] brunneus; Isbrücker \& Nijssen, 1976b: 110 (listed; type-locality; original type-material no longer exists).

Hancock's Loricaria brunnea is a poorly described species. Subsequent material attributed to this species does not possess any of the few characters described, and so is referred to Rineloricaria fallax (see above). The holotype of Loricaria brunnea is apparently lost: an extensive search has been made in the collections of BMNH. Hancock (1828: 240) sent his collection of Demerara animals to the Zoological Society, London, from where they were subsequently transferred to BMNH. Possibly the holotype of Loricaria brunnea (from the Orinoco River) was not amongst this material, and the original description may well have been based on a specimen that was not preserved. On the basis of certain characters mentioned in the original description, we ascribe Loricaria brunnea to the genus Loricariichthys, as it might well prove to be closely related to (or even identical with) Loricariichtbys acutus (Valenciennes, in Cuvier \& Valenciennes, 1840), over which it has priority. It should be added that the holotype of Loricariichtbys acutus is also lost. Only if brown Loricariichtbys specimens from the Orinoco River, reaching a (total or standard) length of 10 to 12 inches (254 to 304.8 $\mathrm{mm}$ ) and with a single row of abdominal scutes will become available, the problem of the identity of Loricaria brunnea can be solved.

C. H. Eigenmann \& R. S. Eigenmann (1889: 37) were unable to identify Loricaria brunnea with any of the subgenera they recognized within Loricaria. In their key of 1890 (: 362 ) L. brunnea is placed into the subgenus Rineloricaria, but evidently this was done inadvertently, as is clear from the separation of the couplets $e$ and $e e$.

Rineloricaria fallax, with which Loricariicbthys brunneus has been often confused, is not known to occur in the Orinoco River drainage.

\section{Rineloricaria hasemani $\mathrm{n}$. $\mathrm{sp}$.}

(Figs. 4, 5a, 6; tables IIa, IIIl-n)

Loricaria (Loricariicbtbys) fallax; Steindachner, 1915: 101102 (in original description; pro parte; “...einem Flusse bei Maguarý an der Braganzabahn bei Pará..." [NMW 44865, ZSM 19732]).

Material examined. -

Holotype: NMW 44865 (paralectotype of Loricaria (Loricariichthys) fallax), sl $136.9 \mathrm{~mm}$, Brazil, Est. Pará, Maguarý [locality not traced] near Belém, along the Bragança railroad [Bragança, $01^{\circ} 02^{\prime} \mathrm{S} 46^{\circ} 46^{\prime} \mathrm{W}$ ], in forest streams which empty into Rio Guamá [Guamá, $01^{\circ} 35^{\prime} \mathrm{S} 47^{\circ} 29^{\prime} \mathrm{W}$ ], coll. J. D. Haseman, 31-VII-1913.

Paratypes: ZSM 19732 (one, ex NMW 44865, paralectotype of Loricaria (Loricariichthys) fallax), sl $116.6 \mathrm{~mm}$, same data as holotype; NMW 45133 (one), sl $103.2 \mathrm{~mm}$, Brazil, Est. Pará, Lema [locality not traced; according to Haseman's unpublished travel diary (p. 24), this is "...nothing 


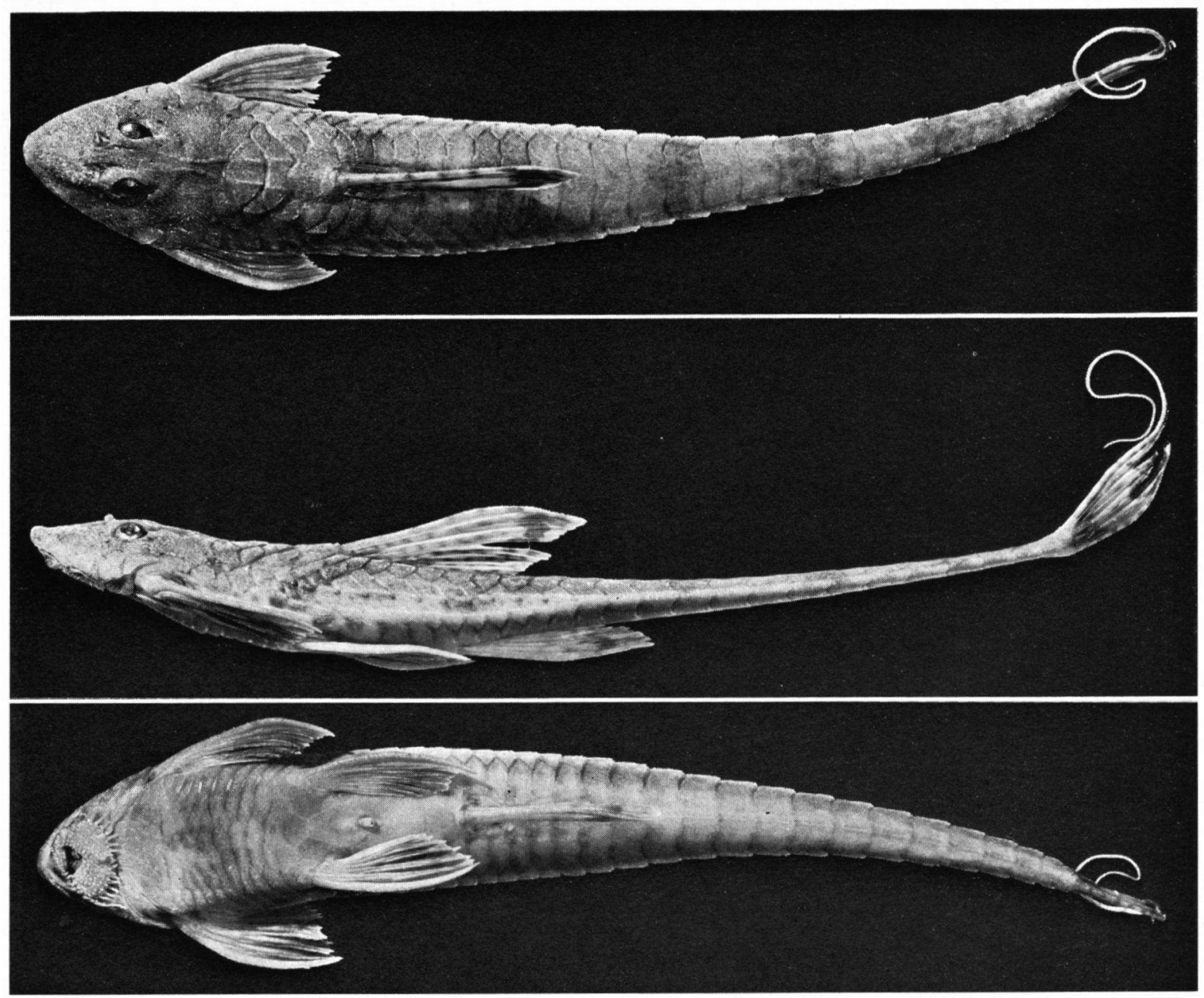

Fig. 4. Rineloricaria basemani n. sp., holotype in dorsal, lateral, and ventral view.

more than a sawmill and rum factory combined in one large building."] near Belém, in a stream flowing into Rio Bem Fica [not traced] which enters the Amazon near Pinheiro [not Pinheiro at $02^{\circ} 31^{\prime} \mathrm{S} 45^{\circ} 05^{\prime} \mathrm{W}$ ], coll. J. D. Haseman, 1-VIII-1913.

\section{Description. -}

Morphometric and meristic data are presented in tables IIa and IIIl-n, and are not repeated here.

In many of its morphological characters, Rineloricaria basemani is similar to $R$. formosa described above, although it seems more closely related to $R$. stewarti. It differs from $R$. formosa in the following characters: Ridges on supraoccipital process diverge less strongly. Tip of supraoccipital process broad and flat. Odontodes somewhat more prominent, especially on the dorsum of the head and anterior part of the body, where they are arranged into fine wavy lines. Teeth almost exactly as in $R$. formosa, except for the shape of the tips, having an acute or slightly roundish lanceolate form (fig. 5a).

Colour in alcohol (fig. 4). - Ground colour of ossified parts brownish tan, dorsal part of head and body darker than ventral part; unossified parts whitish.

Dorsum of body with five rather ill-defined, transverse brown stripes, each with several minute dark brown spots. The first stripe originates at the base of the first to second or third branched dorsal fin ray and extends oblique anteriorly, reaching to the dorsal edge of the thoracic scutes. The second stripe is quite broad (extending over two transverse dorsal scutes) and is situated somewhat 
beyond the origin of the last dorsal fin ray. Pores on dorsum of head almost black, dark brown. Ventral part of the body with a dark, tan to brownish, longitudinal streak, originating posterior to the end of the inner pectoral fin ray, extending to the base of the caudal fin. Ridges on coalescing and lateral body scutes mostly unpigmented.

Dorsal fin spine and rays with some small, irregularly arranged brownish spots, especially near the tip of the rays. A more prominent spot occurs at and near the tip of the first or the first and second ray and adjacent membrane. Dorsum of pectoral and pelvic fins rather dark, sometimes with some inconspicuous brownish spots. Anal fin hyaline with or almost without small brownish stains.

Base of caudal fin brown; upper and lower "spine" with regular small dark brown spots; tips of caudal fin rays and adjacent membrane pigmented with dark brown, forming a solid stripe along the outer margin.

Etymology. - Rineloricaria basemani is named after J. D. Haseman, who assembled perfectly preserved collections of South American freshwater fishes in the early 1900's, including the specimens on which this species is based.

Discussion. - Rineloricaria hasemani appears to have close affinities with Rineloricaria stewarti (C. H. Eigenmann, 1909). We have examined the holotype of this species (FMNH 53330, ex CM 1508, sl $57.3 \mathrm{~mm}$, from Guyana, Chipoo Creek (fig. 6), a tributary of the Rio Ireng between Karakara [not traced] and the Rupununi River [cf. C. H. Eigenmann, 1912: 3], coll. W. Grant), the morphometric and meristic data of which are given in tables IIb and IIIo.

Rineloricaria basemani and $R$. stewarti have almost identical colour patterns. $R$. stewarti has no longitudinal ventral streaks, and fewer spots on the dorsal fin rays than $R$. hasemani. $R$. stewarti has very distinct strigilae (in the present case this does not appear to be a juvenile character) on dorsal and dorsolateral scutes, lacking in the much smoother $R$. hasemani. Since $R$. stewarti is known from small specimens only, we are not sure whether it is a "dwarf" species or not. Boeseman (1976: $167-168$, pls. VI \& VII, table 4, fig. 2 , in key on p. 156) records 57 specimens as Loricaria (Loricariichthys) cf. stewarti from 16 Surinam samples. His largest specimen, indicated in pl. VI as a female, is only $104 \mathrm{~mm}$ in sl. In his key he notes: "...males with enlarged spines on interorbital and occipital areas." Our specimens of Rineloricaria basemani do not show these secondary sexually dimorphic characters.
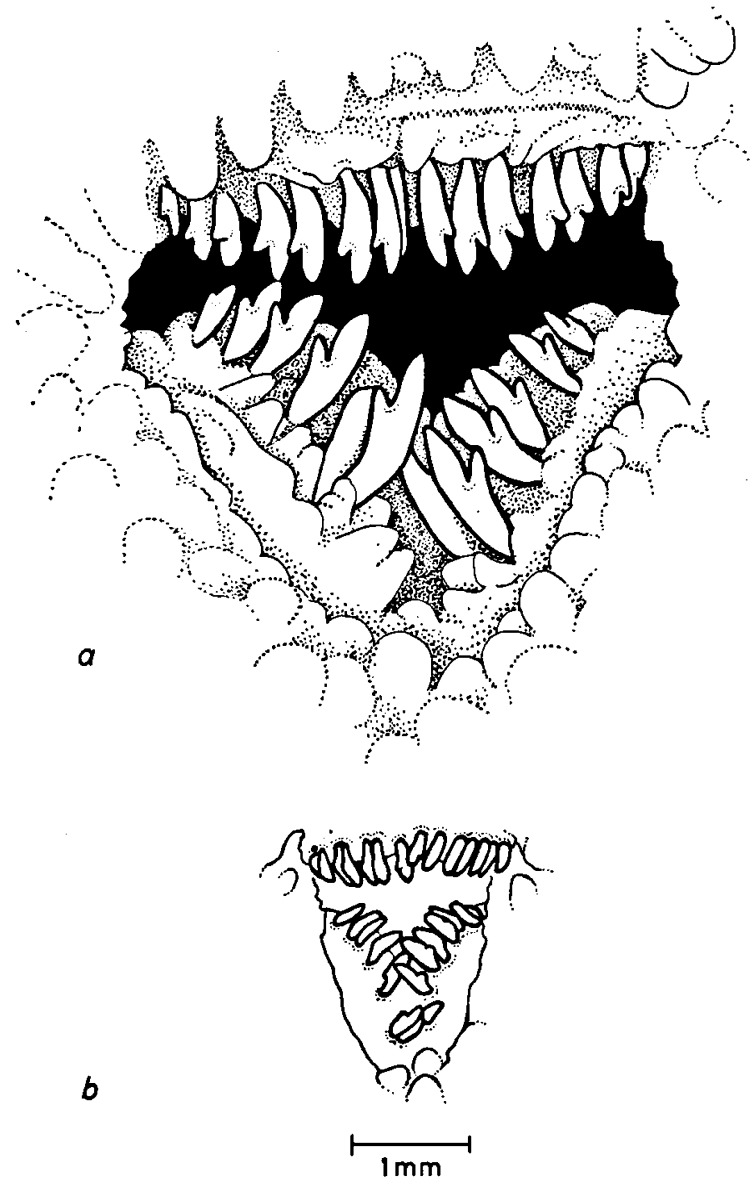

Fig. 5. Dentition of the holotypes of: (a) Rineloricaria basemani n. sp., and (b) Rineloricaria stewarti (C. $\mathrm{H}$. Eigenmann, 1909).

The holotype of Rineloricaria stewarti has teeth (fig. $5 b$ ) which are reminiscent of those in $R$. formosa in shape, but not of those in $R$. basemani. If the holotype of $R$. stewarti only represents some juvenile stage, several morphometric differences found between this species and $R$. basemani (see table III) are likely to disappear when larger specimens of the former become known. There are many differences to be noted: in standard length (and 
axial length), in head length, predorsal length, postanal length, dorsal spine length, first dorsal ray, anal spine length, pectoral spine length, pelvic spine length, lower caudal spine, lower lip, abdominal length, maximum orbital diameter, interorbital width, head width, head depth, body depth at dorsal, body width at anal, depth caudal peduncle, lateral scutes, and in lower jaw teeth.

\section{Loricariichthys platymetopon $\mathrm{n}$. $\mathrm{sp}$.}

(Figs. 6, 7-9; tables IIc, IV, Va-k)

Loricaria maculata (non Bloch, 1794); Valenciennes, in Cuvier \& Valenciennes, 1840: $473-476$ (: $350-351$ in the Strasbourg edition) (description; one specimen [MNHN A.9559] from "...Missions près de l'Ibera-Tingay, dans un marais formé lors des crues par les eaux du Parana"; discussion); Valenciennes, in d'Orbigny, 1847: 8, pl. VI fig. 3 (same specimen as described in 1840); Boulenger, 1896: 33 (listed; Paraguay [BMNH 1895.5.17:102]).

Loricaria typus (non Bleeker, 1862); Regan, 1904: 286-287 (pro parte; description [partly based on BMNH 1895.5.17: 102]); A. de Miranda Ribeiro, 1918: 719 (listed, as no. 75).

Loricariicbtbys maculatus (non Bloch, 1794); Lüling, 1975: 175 , 179, figs. 3-4 (only part of the material examined [ZMA 110.929, ZMA 110.934]; biotope; Argentina, Isla los Sapos).

Material examined. -

Holoty pe: USNM 181580, sl $222.3 \mathrm{~mm}$, male, Paraguay, Lago Ypacarai, San Bernardino, $25^{\circ} 16^{\prime} \mathrm{S} 57^{\circ} 16^{\prime} \mathrm{W}$, Río Paraguay system, coll. C. J. D. Brown, 16-VIII-1956.

$\mathrm{P}$ ar at y pes : USNM 181768 (one), sl $286.8 \mathrm{~mm}$, Paraguay, Asunción Bay, Río Paraguay near Asunción, $25^{\circ} 15^{\prime} \mathrm{S}$ $57^{\circ} 40^{\prime} \mathrm{W}$, coll. C. J. D. Brown, 20-XII-1956; USNM 181755 (two), ZMA 114.326 (one), sl 276.5 to $299 \mathrm{~mm}$, Paraguay, Río Paraguay, Asunción Bay near Asunción, coll. C. J. D. Brown, 8-I-1957; BMNH 1895.5.17:102 sl $141.5 \mathrm{~mm}$, Paraguay, Río Paraguay near Asunción Bay, coll. C. J. D. Brown, 8-I-1957; BMNH 1895.5.17:102 (one), sl $266.5 \mathrm{~mm}$, Río Paraguay, coll. C. Ternetz; BMNH 1927.11.23:38-40 (three), sl 83.3 to $216 \mathrm{~mm}$, \& BMNH 1928.1.6:1-5 (five), sl 73.5 to $87.5 \mathrm{~mm}$, Paraguayan Chaco, coll. G. S. Carter; NMW 45126 (one), sil $297.8 \mathrm{~mm}$, Paraguay, Asunción; ZSM 4830 (one), sl 270 mm, Paraguay, Asunción, don. F. Steindachner, collected in 1909; NMW 45128 \& NMW 45073 (two), sl 229 and $257 \mathrm{~mm}$, Paraguay, Hapitapunta or Itapitapunta [locality not traced], coll. C. Ternetz.

MNHN A.9559 (one), sl 235.4 mm, Argentina, Río Paraná, coll. A. d'Orbigny; MACN no register number (one), sl $249.3 \mathrm{~mm}$, Argentina, Prov. Santa Fé, Río Colastiné, coll. López, Castello \& Ehrlich, 26/30-VII-1975; ZMA 110.929 (six), sl 112.6 to $186 \mathrm{~mm}$, Argentina, tributary of Isla de llos Sapos, middle course of Río Paraná, coll. K. H. Lüling, 3-XII1970; ZMA 110.934 (one), sl 275 mm, Argentina, Laguna la Quinta, middle course of Río Paraná, coll. K. H. Lüling, 10-XII-1970.

MZUSP 970 (two) (register number pro parte), sl 165.5 and $196 \mathrm{~mm}$, males, Brazil, Est. Grande do Sul, Rio Uruguay at Itaqui, coll. E. Garbe, 1914.
MZUSP 14100 (onc), sl $262 \mathrm{~mm}$, male, Brazil, Est. Amazonas, channel between Lago Janauaca and Rio Solimões, approximately $03^{\circ} 25^{\prime} \mathrm{S} 61^{\circ} 21^{\prime} \mathrm{W}$, coll. R. V. "Alpha Helix", carly XII-1976.

Nontypes: ZFMK I/70-71/3354 through 3359, ZFMK I/70-71/3386 through 3398 (nineteen), sl 108.8 to $180.2 \mathrm{~mm}$, Argentina, Isla de los Sapos, coll. K. H. Lüling, 2/3-XII-1970 (material may be found to be polyspecific); ZMA 114.350 (two), sl 250 and $288 \mathrm{~mm}$, Argentina, aquarium specimens without locality data, gift from BMNH.

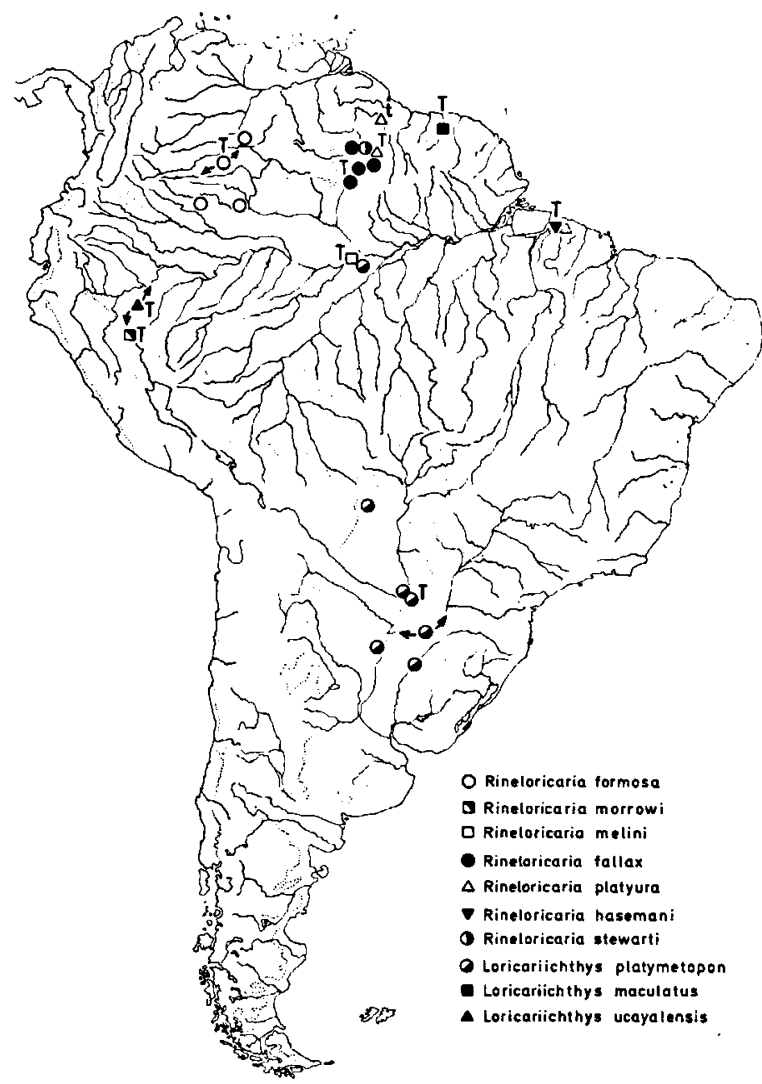

Fig. 6. Collecting localities of Rineloricaria formosa n. sp., $R$. morrowi Fowler, 1940, $R$. melini (Schindler, 1959), $R$. fallax (Steindachner, 1915), $R$. platyura (Müller \& Troschel, in Schomburgk, 1848), $R$. hasemani n. sp., $R$. stewarti (C. H. Eigenmann, 1909), Loricariichthys platymetopon n. sp., L. maculatus (Bloch, 1794) (type-locality only), and of L. ucayalensis Regan, 1913; except for L. maculatus, the symbols used are based on the specimens examined for the present publication. The type-localities are indicated by $\mathrm{T}$; $t$ indicates the type-locality of Loricaria submarginatus (= Rineloricaria platyura) arrows indicate localities we could not trace more precisely.

Description. -

Morphometric and meristic data are presented in tables IIc, IV and Va-k, and are not repeated here.

A posterior, a middle, and an anterior complex of abdominal scutes can be distinguished: 
(1) The posterior complex consists of a welldeveloped anal plate, anteriorly bordered by a small number of rather large, polygonal scutes which, together with the posterior thoracic scute, form a large, inflexible preanal plate. The anal and preanal plates are a fused continuation of the laterally movable ventral scutes of the caudal peduncle.

(2) The middle complex has, together with the well-developed adjoining thoracic scutes, a function in allowing up and down movements of the head; these movements are possible only because of the presence of this complex and the movable scutes anterior to the origin of the dorsal fin spine and posterior to the supraoccipital process.

Principally three midventral transverse "series" of scutes are present anterior to the preanal plate. Usually the posterior scute is single, whereas the two series anterior to this scute consist of two or three parts irregularly fused somewhere in the middle.

(3) The anterior complex of scutes reaches to. about the height of the origin of the last pectoral fin ray. It is a quite firm, inflexible, anteriorly rounded plate, consisting of smaller polygonal scutes, decreasing in size anteriorly.

A comparatively large, triangular plate is present anterior to the branchiostegal membrane. It is an isolated extension of the ventrally ossified head margin. All dermal ossifications including scutes, fin spines, and rays are covered with numerous minute odontodes giving a quite smooth appearance. Contrary to the condition found in Rineloricaria beteroptera Isbrücker \& Nijssen, 1976, these odontodes are not sexually dimorphic. The odontodes are prominently larger in two rows along the coalescing and parallel lateral body scutes.

A conspicuous arbital notch is present, its dorsal margin is rounded posteriorly.

Anteriorly the head has a rather broad, naked margin which narrows towards the opercular region, reaching to about the height of the posterior point of the orbital notch.

A minute pectoral pore is present just ventral to the posteroventral tip of the cleithrum. There are simple pores of the sensory canal system on the dorsum of the head and snout, and on the ventrally ossified snout margin, and bifurcate pores between the two rows of prominent odontodes along the lateral body scutes. Those pores between the coalescing scutes are situated just below the posterior edges of the dorsal row.

Secondary sexually dimorphic characters are found as differences in structure and pigmentation of the lips (compare fig. 7, a male, with fig. 8 , a female), and in shape of the teeth.

In the female, the dorsal side of the upper lip consists of smooth skin extending from the ventral tip of the snout to just anterior of the upper jaws, then running laterally along part of the rictal barbels, its edge becoming papillate with about ten to eighteen subbarbels. The ventral side of the upper lip is covered with numerous weakly developed soft striae, and ridges of papillae; larger papillae are situated near the subbarbels just mentioned. The edges on either side of the upper jaw are almost separated from each other by a small, triangular flap of skin originating just anterior to the symphysis of the upper jaws. On the ventral surface of this flap there is a medial ridge of skin, running to a point between the right and left upper jaws.

The upper and lower lips are connected with each other by rictal barbels, together forming a sort of sucking device. The lower lip consists of two thick, cushion-like parts - thickest towards the buccal cavity - with a median separation bridged by thin skin. This surface is covered anteriorly with rather inconspicuous soft striae and ridges of weak papillae, increasing in size towards its edge. A more or less prominent median notch may be present. The edge of the lower lip has small triangular papillae and minute flaps.

In mature males the lower lip and posterior part of the upper lip become gradually longer and broader, to form a huge labial velum. During this process the two cushion-like structures present in females and juveniles disappear completely. This labial velum has an important function in the protection of eggs (and possibly of larval stages). A male in ZMA 110.929 with a sl of $186 \mathrm{~mm}$ has a lower lip length of $36.3 \mathrm{~mm}$ and, measured between the points where the rictal barbels project from the lips, a width of about $53 \mathrm{~mm}$.

In the buccal cavity, posterior to the upper jaws 

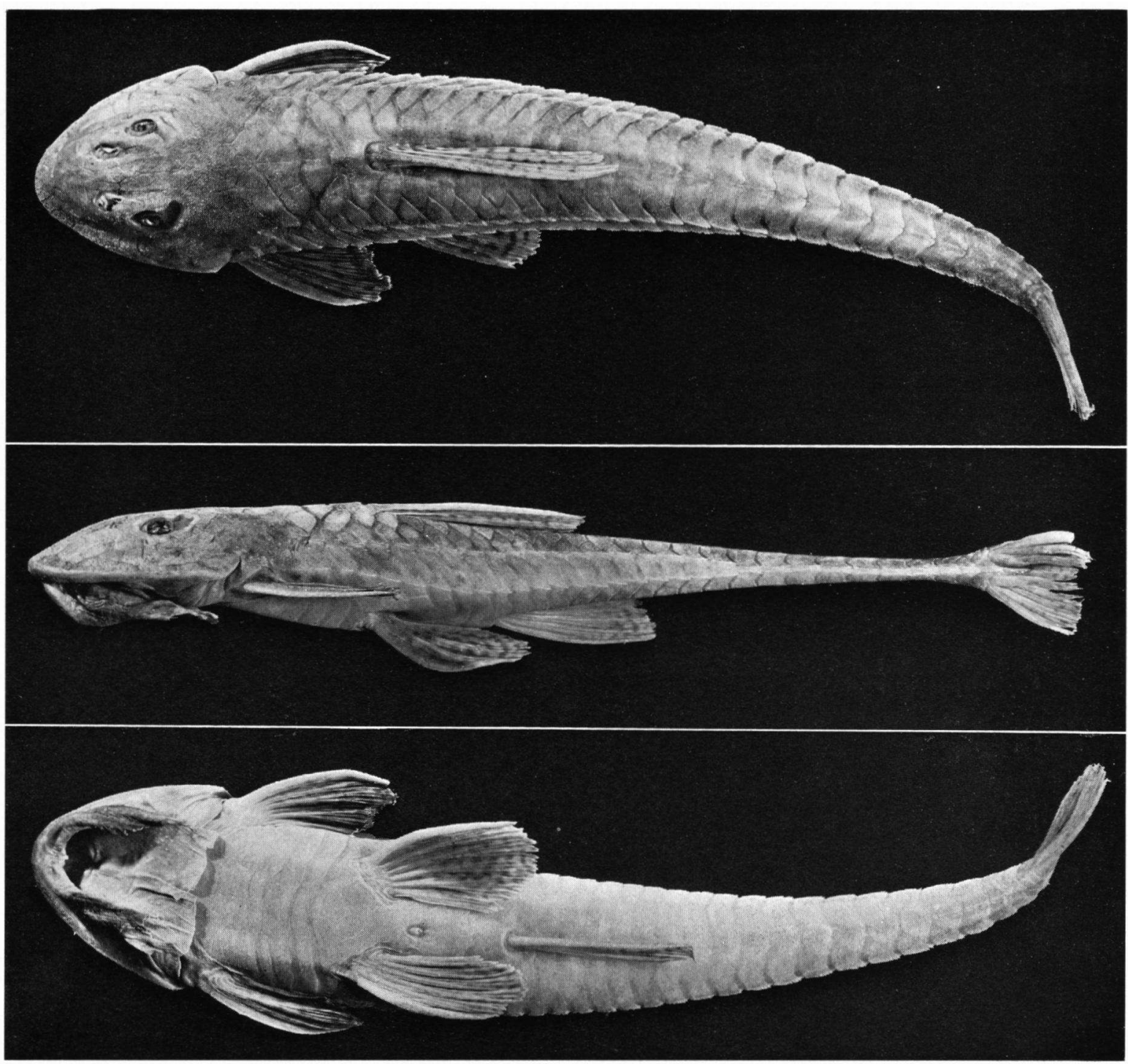

Fig. 7. Loricariichtbys platymetopon n. sp., holotype (male) in dorsal, lateral, and ventral view.

are two narrow transverse flaps of skin with roundish outer edges, connected in the middle. The outer surface of these flaps is provided with a number of elongate axial ridges and flat papillae.

Inconspicuous bilobed teeth (inner lobe larger than outer lobe) are present in both upper and lower jaws; they are about equal in length in both jaws; however, the outer teeth are somewhat smaller than the inner ones. In females and juveniles the tips of the teeth are slender and acute, in nuptial males the tips become much broader and rounded.

Tip of supraoccipital process rather acute.
Eye with a narrow dorsal covering of blackish and silvery pigmented skin, the iris partly covered by a small, rounded or triangular dorsal flap.

Colour in alcohol (figs. 7-8). - Ground colour of dorsum of the fish (up to ventral row of prominent lateral odontodes) uneven tan and greyish tan. In some specimens, including the holotype, there is a more or less faint concentration of dark brown pigment on the dorsum of the head, forming small, irregular blotches. In the paratype in MACN these blotches tend to form small, more or less vermic- 


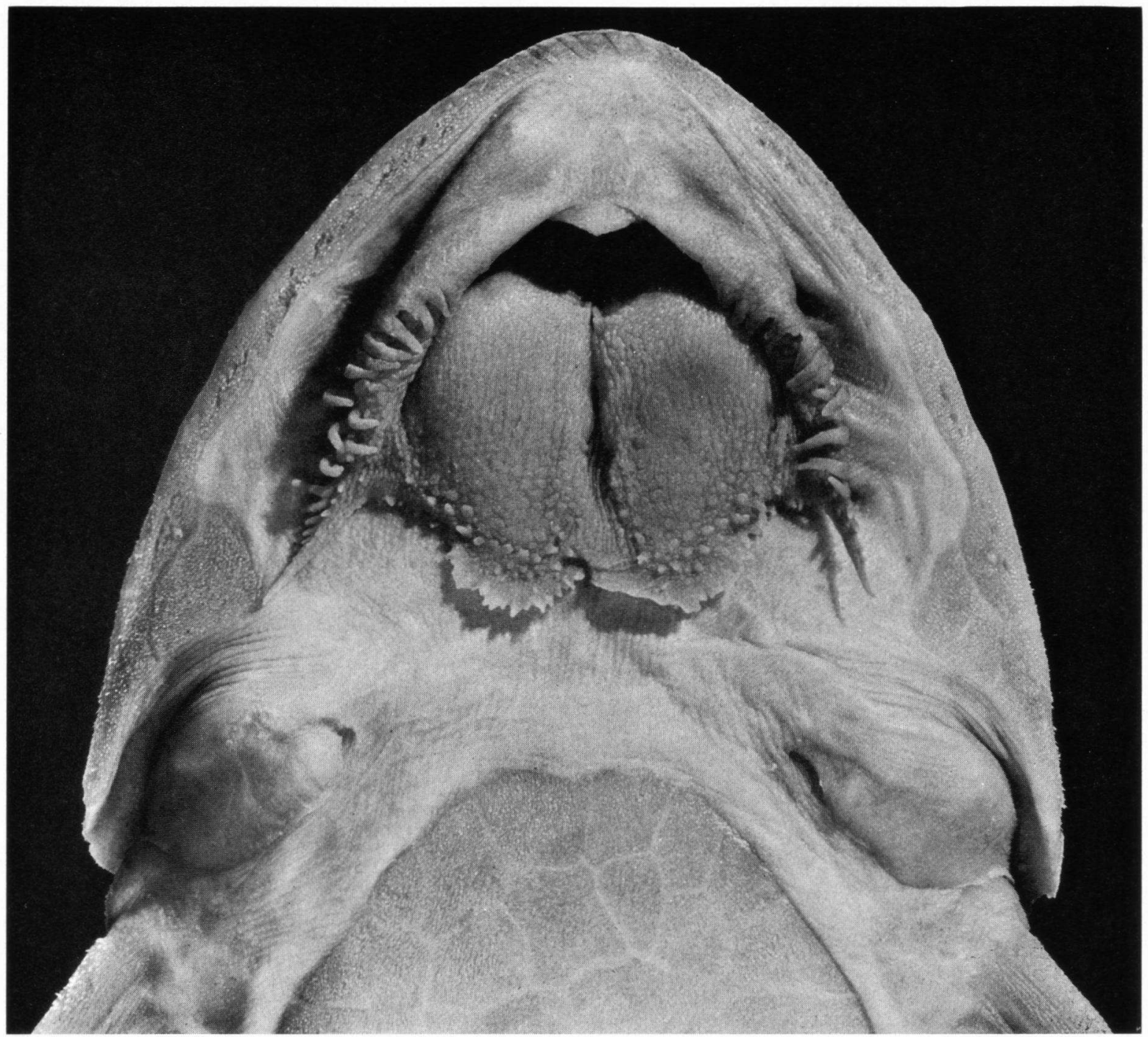

Fig. 8. Loricariicbtbys platymetopon n. sp., detail of a paratype (female, USNM 181755, sl $276.5 \mathrm{~mm}$ ), anterior part in ventral view, showing shape and structure of the lips.

ular lines; some ill-defined, widely spread darker markings occur on the dorsum of the body and between the coalescing lateral odontode ridges.

Ventral part of the body light tan, without pigmentation.

All fins generally with dark brown spots. Dorsal fin spine, rays and membrane with an irregular number of distinct roundish or square spots and blotches which tend to be arranged into oblique series.

Caudal fin with numerous small spots which seem to be arranged into irregular vertical series. In some specimens the lower caudal fin lobe has concentrated dark brown pigment forming a faint blotch about the posterior end of the two ventral branched rays.

Anal fin in some specimens with a few small, widely scattered spots. In other specimens the anal fin is provided with numerous small, well-defined spots which may tend to form up to about seven oblique or roundish series.

Dorsum of pectoral and pelvic fins usually with conspicuous irregular blotches, forming oblique or roundish series. In some specimens the dorsum of these two pairs of fins is provided with irregular, heavy concentrations of blackish pigment giving the appearance of marble.

Laterodorsal side of the lips often with some 
uneven dark greyish-brown pigment, especially in nuptial males. In these males the pectoral fin also often has a somewhat darker dorsal part.

Juveniles. Seven of the paratypes (two in BMNH 1927.11.23:38-40, five in BMNH 1928.1.6:1-5) range from 73.5 to $90.3 \mathrm{~mm}$ sl. Even the smallest specimen has the abdominal scutes completely developed. Because of some morphometric differences (e.g., their narrower interorbital, clearly an allometric feature in this species), these small specimens are termed juveniles, also because of the following peculiarities. The posterior orbital notch is distinctly weaker developed and lacks the characteristic shape which gradually develops with age. Sensory pores show up more clearly, especially along the dorsal snout margin and the ventrally ossified snout margin, causing a more coarse appearance of these parts than found in larger specimens. The colour pattern in these small specimens is not pronounced, at least in preserved state.

Etymology. - The specific name platymetopon is from the Greek $\pi \lambda \alpha \tau$ 's meaning broad, and from the Greek $\mu \varepsilon$ é $\omega \pi$ ov meaning forehead, an allusion to the broad interorbital area, which is diagnostic for this species.

Discussion. - Loricariichthys platymetopon has been described under various names, at least since 1840: Valenciennes's description of Loricaria maculata was based on a specimen we designate as a paratype of Loricariichthys platymetopon, just like the specimen recorded as Loricaria maculata from Paraguay by Boulenger (1896). The latter speci-

\section{TABLE IV}

Morphometric and meristic data of 16 specimens of Loricariichthys platymetopon n. sp. from Paraguay: (a) holotype, (b-p), paratypes, as follows: (b) USNM 181755, (c) NMW 45126, (d) ZMA 114.326, (e) USNM 181768, (f) USNM 181755, (g) BMNH 1895.5.17:102, (h) NMW 45073, (i) NMW 45128, (j, 1, and o) BMNH 1927.11.23:38-40, (k) USNM 181437, (m, n, and p) BMNH 1928.1.6:1-5.

\begin{tabular}{|c|c|c|c|c|c|c|c|c|c|c|c|c|c|c|c|c|}
\hline specimen & a & $b$ & c & d & $\mathbf{e}$ & f & g & $\mathbf{h}$ & i & j & $\mathbf{k}$ & 1 & m & n & 0 & p \\
\hline standard length & 222.3 & 299.0 & 297.8 & 288.8 & 286.8 & 276.5 & 266.5 & 257.0 & 229.0 & 216.0 & 141.5 & 90.3 & 87.5 & 86.1 & 83.3 & 80.1 \\
\hline axial length & 246.1 & 328.2 & - & 316.7 & 317.7 & 303.5 & - & 286.0 & 251.7 & 240.9 & 155.1 & - & 96.8 & 96.8 & 92.8 & 90.1 \\
\hline total length & - & - & - & - & - & - & $>309.6$ & - & - & - & - & $>109.9$ & - & \pm 117.8 & - & $>101.4$ \\
\hline head length & 4.3 & 4.4 & 4.3 & 4.4 & 4.4 & 4.4 & 4.4 & 4.5 & 4.4 & 4.1 & 4.4 & 4.5 & 4.3 & 4.2 & 4.4 & 4.0 \\
\hline predorsal length & 3.0 & 2.9 & 2.9 & 3.0 & 3.0 & 2.9 & 2.9 & 3.0 & 3.1 & 2.8 & 3.1 & 3.1 & 3.0 & 3.0 & 3.1 & 3.1 \\
\hline postdorsal length & 1.8 & 1.8 & 1.8 & 1.8 & 1.8 & 1.8 & 1.8 & 1.8 & 1.9 & 1.9 & 1.7 & 1.7 & 1.8 & 1.8 & 1.8 & - \\
\hline postanal length & 2.0 & 2.1 & 2.2 & 2.1 & 2.1 & 2.1 & .2 .1 & 2.1 & 2.2 & 2.2 & 2.0 & 2.0 & 2.1 & 2.0 & 2.0 & - \\
\hline dorsal spine length & 4.5 & - & 4.4 & - & $<5.9$ & $<5.4$ & 4.1 & - & 4.4 & - & - & 4.1 & 4.1 & 3.8 & 4.0 & - \\
\hline first dorsal ray & 4.6 & - & 4.8 & - & $<5.4$ & $<5.4$ & 4.4 & $<4.8$ & 5.0 & 4.4 & 5.0 & 4.3 & 4.2 & 3.9 & 4.2 & - \\
\hline anal spine length & 5.6 & 6.2 & 5.9 & 6.5 & 5.8 & 6.0 & 6.0 & 5.5 & 6.3 & 5.1 & 5.7 & $5: 4$ & 5.5 & 5.3 & 5.3 & - \\
\hline pectoral spine length & 6.2 & 6.8 & 6.4 & 7.0 & 6.3 & 6.4 & 6.9 & 6.5 & 7.1 & 6.1 & 7.2 & 6.6 & 6.4 & 5.5 & 6.5 & - \\
\hline pelvic spine length & 6.7 & 7.4 & 6.9 & 7.8 & 6.9 & 7.1 & 7.4 & 6.2 & 6.9 & 6.0 & 6.7 & 6.1 & 5.8 & 5.6 & 6.3 & - \\
\hline upper caudal spine & - & - & - & - & - & $<6.5$ & $<6.1$ & - & - & - & - & $<4.5$ & - & \pm 2.7 & - & $<3.8$ \\
\hline lower caudal spine & $\cdots$ & - & 8.5 & - & - & - & 7.7 & 6.4 & 8.2 & - & 7.3 & 6.5 & 6.1 & 5.7 & - & 5.8 \\
\hline snout length & 2.0 & 2.0 & 2.0 & 2.1 & 2.1 & 2.0 & 2.1 & 2.0 & 2.1 & 2.1 & 2.1 & 2.3 & 2.2 & 2.2 & 2.3 & 2.3 \\
\hline lower lip & 2.0 & 3.2 & 4.6 & 3.4 & 3.4 & 3.6 & 3.8 & 4.1 & 1.9 & 3.0 & 3.7 & 4.1 & 3.6 & 3.2 & 3.8 & - \\
\hline thoracic length & 1.5 & 1.6 & 1.5 & 1.5 & 1.3 & 1.5 & 1.5 & 1.5 & 1.6 & 1.4 & 1.4 & 1.4 & 1.5 & 1.5 & 1.4 & - \\
\hline abdominal length & 1.6 & 1.5 & 1.5 & 1.4 & 1.4 & 1.5 & 1.5 & 1.4 & 1.6 & 1.6 & 1.6 & 1.6 & 1.5 & 1.6 & 1.6 & - \\
\hline max. orbital diameter & 4.3 & 4.0 & 4.6 & 4.6 & 4.3 & 4.2 & 4.3 & 4.3 & 4.0 & 4.2 & 4.0 & 3.7 & 3.9 & 3.9 & 3.9 & 4.0 \\
\hline interorbital width & 3.3 & 3.2 & 3.2 & 3.4 & 3.3 & 3.3 & 3.5 & 3.1 & 3.5 & 3.4 & 3.9 & 3.9 & 4.0 & 4.1 & 4.1 & 4.5 \\
\hline cleithral width & 1.2 & 1.2 & 1.2 & 1.3 & 1.2 & 1.3 & 1.3 & 1.1 & 1.3 & 1.2 & 1.3 & 1.2 & 1,3 & 1.3 & 1.3 & - \\
\hline supra-cleithral width & 1.5 & 1.5 & 1.5 & 1.6 & 1.5 & 1.6 & 1.7 & 1.5 & 1.7 & 1.6 & 1.7 & 1.7 & 1.6 & 1.7 & 1.7 & - \\
\hline head width & 1.2 & 1.2 & 1.2 & 1.3 & 1.2 & 1.3 & 1.3 & 1.1 & 1.3 & 1.2 & 1.3 & 1.2 & 1.3 & 1.3 & 1.3 & - \\
\hline head depth & 2.2 & 2.1 & 2.3 & 2.1 & 2.1 & 2.2 & 2.3 & 2.2 & 2.5 & 2.0 & 2.5 & 2.3 & 2.4 & 2.5 & 2.4 & - \\
\hline body depth at dorsal & 1.8 & 1.9 & 2.0 & 2.0 & 1.8 & 2.0 & 2.0 & 1.9 & 2.2 & 1.7 & 2.1 & 2.0 & 2.0 & 2.2 & 2.0 & - \\
\hline body width at dorsal & 1.4 & 1.4 & 1.3 & 1.5 & 1.3 & 1.4 & 1.5 & 1.4 & 1.6 & 1.3 & 1.6 & 1.5 & 1.6 & 1.6 & 1.6 & 1.6 \\
\hline body width at anal & 1.7 & 1.7 & 1.6 & 1.8 & 1.6 & 1.7 & 1.7 & 1.6 & 1.9 & 1.6 & 1.8 & 1.7 & 1.8 & 1.9 & 1.8 & 1.9 \\
\hline depth caudal peduncle & 7.6 & 7.7 & 8.9 & 7.6 & 7.3 & 7.0 & 7.3 & 9.2 & 10.7 & 6.6 & 8.6 & 11.1 & 11.9 & 12.8 & 11.2 & 13.2 \\
\hline width caudal peduncle & 6.0 & 5.9 & 5.4 & 6.3 & 6.0 & 5.7 & 5.6 & 5.3 & 6.3 & 5.6 & 6.9 & 6.2 & 6.2 & 6.2 & 6.2 & - \\
\hline rictal barbel & 2.1 & 3.2 & 2.6 & 2.9 & 2.9 & 2.7 & 3.1 & 2.5 & - & 2.4 & 2.6 & 2.5 & 2.4 & 2.3 & 2.8 & - \\
\hline latersl scutes & $32 / 32$ & $32 / 32$ & $32 / 32$ & $32 / 32$ & $32 / 32$ & $32 / 32$ & $32 / 32$ & $31 / 32$ & $32 / 32$ & $32 / 32$ & $33 / 32$ & $32 / 32$ & $32 / 32$ & $30 / 31$ & $32 / 32$ & $32 / 32$ \\
\hline coalescing scutes & $21 / 21$ & $20 / 21$ & $21 / 21$ & $20 / 20$ & $21 / 21$ & $21 / 21$ & $21 / 20$ & $20 / 20$ & $21 / 21$ & $20 / 20$ & $21 / 21$ & $20 / 20$ & $20 / 21$ & $20 / 20$ & $20 / 20$ & $21 / 20$ \\
\hline thoracic scutes & $7 / 7$ & $7 / 7$ & $6 / 6$ & $7 / 7$ & $7 / 7$ & $7 / 7$ & $7 / 6$ & $6 / 6$ & $5 / 6$ & $7 / 8$ & $7 / 5$ & $7 / 7$ & $7 / 7$ & $5 / 6$ & $7 / 7$ & $6 / 7$ \\
\hline teeth upper jaws & $12 / 10$ & $11 / 17$ & $15 / 13$ & $12 / 15$ & $10 / 10$ & $10 / 12$ & $16 / 14$ & $14 / 13$ & $12 / 10$ & $11 / 12$ & $8 / 10$ & $7 / 7$ & $7 / 7$ & $6 / 6$ & $5 / 7$ & $10 / 9$ \\
\hline teeth Iower jaws & $15 / 14$ & $18 / 19$ & $14 / 16$ & $25 / 20$ & $11 / 11$ & $15 / 11$ & $25 / 24$ & $21 / 19$ & $19 / 17$ & $20 / 18$ & $8 /-$ & $8 / 8$ & $9 / 9$ & $8 / 8$ & $8 / 8$ & $12 / 15$ \\
\hline
\end{tabular}


men was subsequently identified as Loricaria typus (within the subgenus Loricariicbtbys) by Regan (1904). Only some of the specimens recorded erroneously as Loricariichthys maculatus by Lüling (1975) are identified with certainty as Loricariichthys platymetopon, being the only specimens of his numerous samples we were able to examine.

Recently, the holotype of Parabemiodon typus Bleeker, 1862 (RMNH 3121; type-species of the genus Parabemiodon Bleeker, 1862) was received on loan (cf. Isbrücker \& Nijssen, 1976b: 110). It has been compared directly with the lectotype of Loricaria maculata Bloch, 1794 (ZMB 3163; type-species of the genus Loricariichthys Bleeker, 1862). Most of the few differences between these two specimens (see tables IId-e, and VI-n) seem to lie within the range of phenotypic variation, and indicate that Parabemiodon typus should be considered a junior synonym of Loricariichthys maculatus. This would confirm the opinion expressed by Van der Stigchel (1946 \& 1947: 172-174) on this part of his synonymy of Loricaria maculata, supported by Boeseman's recent (1976: 160-165) account on Loricaria (Loricariichtbys) maculata, in which he includes a re-examination of the holotype of Parabemiodon typus. However, there is one character in which this holotype differs from other, comparable, Surinam specimens: its wide interorbital (according to column $\mathrm{S}$ in table 2 of Boeseman, 1976: 162, it is $4.15 \mathrm{in} \mathrm{hl}$ against 4.30 to 5.25 in 30 other specimens ). It is not surprising that Loricariichtbys platymetopon has sometimes been identified as Parabemiodon typus. Most representatives of the genus Loricariichthys have a much narrower interorbital. Bleeker (1864, pl. XIII fig. 1) published an illustration of the predorsal region of the holotype of Parabemiodon typus, showing the interorbital area slightly broader (about 3.8 in head length, agreeing with some of the paratypes of $L$. platymetopon with a comparable sl) than is actually the case ( $4.1 \mathrm{in} \mathrm{hl}$, same as in the lectotype of Loricariichtbys ucayalensis Regan, 1913).

Loricariichthys maculatus has been recorded from several localities, including specimens originating from localities where $L$. platymetopon were found; the same holds true for records of Parabemiodon typus. At least some of these speci- mens might prove to be conspecific with our new species. We suspect that most records of Parabemiodon typus from at least the Río Paraná system actually represent Loricariichthys platymetopon. For example, in Ringuelet et al. the species keying out (1967: 405) as Loricaria (Loricariichtbys) typus is probably identical with $L$. platymetopon. However, several different species of Loricariichtbys occur in the Río Paraná and Río Paraguay system, and we prefer to examine all this material before assigning the various and numerous references to any synonymy.

The occurrence of Loricariicbthys platymetopon in Venezuela seems likely because of records of L. typus (e.g., Machado Allison \& López Rojas, 1975: 93-119). These authors published an excellent account on developmental stages of a Loricariicbtbys they identified as L. typus, originating from "...cerca de Camaguán [08 $\left.09^{\circ} \mathrm{N} 67^{\circ} 37^{\prime} \mathrm{W}\right]$, puerto sobre el río Portuguesa, Estado Guárico, Venezuela.", Orinoco River drainage. It would be necessary to compare all their material (as well as specimens previously identified from Venezuela as typus and maculatus, e.g., by Pellegrin, 1899: 158, Rio Apure; Schultz, 1949: 77, Caripito; Fowler, 1954: 104-105; and by Mago Leccia, 1970: 85 ) before it can be satisfactorily assigned to any species.

Loricariicbthys platymetopon appears to be closely related to Loricariichtbys ucayalensis Regan, 1913, which itself is closely related to Loricariichthys maculatus (Bloch, 1794) (including Parabemiodon typus). L. ucayalensis was originally described from two syntypes, both nuptial males. We herewith designate the larger as the lectotype (BMNH 1913.7.30:36, sl $198 \mathrm{~mm}$ ). The only paralectotype (BMNH 1913.7.30:37) is $171 \mathrm{~mm}$ in sl (tables IIf, Vo-p). L. ucayalensis and $L$. maculatus eventually might prove to deserve distinction at subspecific rather than at specific level, or even may have to be regarded not distinct at all. $L$. platymetopon differs from both $L$. ucayalensis and $L$. maculatus mainly in three characters: it attains a larger standard length (up to $299 \mathrm{~mm}$ against 198 and $212.5 \mathrm{~mm}$ for L. ucayalensis and L. maculatus, respectively), it has a more strikingly spotted colour pattern than both L. ucayalensis and L. maculatus, and it is characterized by a broader 
TABLE V

Morphometric and meristic data of (a-k) 11 paratypes of Loricariichthys platymetopon n. sp., from Paraguay (a-b) and from Argentina (c-k), as follows: (a-b) BMNH 1928.1.6:1-5, (c) ZMA 110.934, (d) MACN no register number, (e) MNHN A.9559, (f-k) ZMA 110.929, (1) Loricariichthys maculatus (Bloch, 1794), lectotype, (m) Loricariicbthys maculatus, paralectotype, (n) Loricariichthys maculatus, holotype of Parabemiodon typus Bleeker, 1862, (o) Loricariichthys ucayalensis Regan, 1913, lectotype, and (p) Loricariichthys ucayalensis, paralectotype. An asterisk indicates correction of previously published data (Isbrücker, 1971: 12-13) of these two specimens, which were re-examined for the present study.

\begin{tabular}{|c|c|c|c|c|c|c|c|c|c|c|c|c|c|c|c|c|}
\hline specimen & a & b & c & d & e & f & g & h & $\mathbf{i}$ & $\mathbf{j}$ & k & 1 & m & n & 0 & $\mathbf{p}$ \\
\hline standard length & 75.0 & 73.5 & 275.0 & 249.3 & 235.4 & 186.0 & 158.0 & 152.0 & 134.9 & 133.1 & 112.3 & $12.5^{*}$ & 109.0 & 177.0 & 198.0 & 171.0 \\
\hline axial length & 83.9 & 81.9 & 305.7 & - & 261.4 & 206.7 & 175.8 & 177.8 & 151.2 & 148.2 & 123.9 & - & 119.5 & - & 217.0 & 185.0 \\
\hline total length & 95.3 & 92.8 & - & - & - & - & - & - & - & 171.1 & 145.6 & - & - & - & 230.9 & 197.0 \\
\hline head length & 4.3 & 4.4 & 4.2 & 4.2 & 4.3 & 3.9 & 4.4 & 4.5 & 4.2 & 4.3 & 4.4 & $4.9^{\circ}$ & 4.9 & 4.8 & 4.2 & 4.2 \\
\hline predorsal length & 3.1 & 3.2 & 2.9 & 2.9 & 2.9 & 2.7 & 3.0 & 3.0 & 2.9 & 3.0 & 3.1 & 3.2 & $3.4^{*}$ & 3.2 & 2.9 & 2.9 \\
\hline postdorsal length & - & 1.7 & 1.9 & 1.9 & 2.2 & 1.9 & 1.8 & 1.8 & 1.8 & 1.8 & 1.7 & 1.6 & 1.6 & 1.7 & 1.8 & 1.8 \\
\hline postanal length & - & 2.0 & 2.2 & 2.1 & 2.1 & 2.2 & 2.1 & 2.0 & 2.2 & 2.0 & 2.0 & 1.9 & $1.8^{*}$ & 1.9 & 2.1 & 2.1 \\
\hline dorsal spine length & - & 4.0 & 4.3 & 4.8 & - & $<4.0$ & $<4.1$ & $<4.5$ & 3.7 & 3.9 & 4.3 & 4.9 & $4.3^{*}$ & - & 4.7 & 4.6 \\
\hline first dorsal ray & - & 4.2 & 4.4 & 4.7 & - & 4.1 & 4.1 & 4.8 & 3.8 & 4.0 & 4.4 & 5.0 & 4.4 & - & 4.9 & 4.9 \\
\hline anal spine length & - & 5.5 & 5.3 & 5.5 & - & 5.2 & 5.2 & 5.5 & 4.9 & 4.9 & 5.3 & $5.9^{*}$ & $5.9^{*}$ & - & 5.3 & 5.6 \\
\hline pectorsl spine length & - & 6.4 & 6.0 & 6.9 & 6.4 & 5.7 & 6.0 & 6.5 & 6.1 & 6.0 & 6.7 & $6.7^{*}$ & $7.3^{*}$ & - & 6.1 & 6.8 \\
\hline pelvic spine length & - & 6.3 & 6.2 & 6.4 & 6.2 & 5.8 & 5.7 & 6.2 & 5.9 & 6.1 & 6.3 & $5.9=$ & $6.6^{\circ}$ & \pm 6.0 & 6.2 & 9.4 \\
\hline upper caudal spine & $<3.7$ & 3.8 & - & - & - & - & - & - & - & 3.5 & 3.3 & - & - & - & $<5.9$ & - \\
\hline lower caudal spine & 6.0 & 6.2 & - & 7.6 & - & 5.9 & 5.8 & 6.0 & - & 5.7 & 6.1 & - & $7.0^{\circ}$ & - & 6.8 & 6.8 \\
\hline snout length & 2.2 & 2.3 & 2.0 & 2.1 & 2.0 & 2.1 & 2.2 & 2.2 & 2.2 & 2.2 & 2.3 & 2.0 & 2.2 & 1.9 & 1.8 & 2.0 \\
\hline lower lip & - & 3.6 & 1.8 & 3.6 & \pm 1.9 & 1.3 & 2.6 & 4.1 & 3.8 & 3.0 & 4.6 & $2.1^{*}$ & $4.1^{\circ}$ & 1.9 & 1.5 & 1.8 \\
\hline thoracic length & - & 1.4 & 1.4 & 1.6 & 1.5 & 1.6 & 1.3 & 1.4 & 1.4 & 1.5 & 1.5 & $1.4^{*}$ & $1.5^{*}$ & 1.4 & 1.5 & 1.5 \\
\hline abdominal length & - & 1.6 & 1.5 & 1.4 & 1.6 & 1.6 & 1.6 & 1.6 & 1.5 & 1.5 & 1.6 & $1.5^{\circ}$ & 1.5 & 1.5 & 1.7 & 1.8 \\
\hline max. orbital diameter & 3.7 & 3.8 & 4.1 & 4.4 & 4.0 & 4.2 & 4.0 & 3.9 & 4.0 & 3.9 & 3.9 & $4.3^{\circ}$ & $3.8^{\circ}$ & 4.0 & 4.3 & 4.7 \\
\hline interorbital width & 4.2 & 4.4 & 3.3 & 3.5 & 3.3 & 3.8 & 3.5 & 3.6 & 3.8 & 3.9 & 3.9 & 4.5 & 4.8 & 4.1 & 4.1 & 4.5 \\
\hline cleithrs? width & - & 1.3 & 1.2 & 1.2 & 1.3 & 1.3 & 1.2 & 1.2 & 1.2 & 1.2 & 1.2 & 1.4 & 1.3 & 1.3 & 1.3 & 1.4 \\
\hline supra-cleithral width & - & 1.7 & 1.5 & 1.6 & 1.6 & 1.7 & 1.6 & 1.6 & 1.6 & 1.7 & 1.6 & 1.7 & 1.7 & 1.6 & 1.6 & 1.8 \\
\hline head width & - & 1.3 & 1.2 & 1.2 & 1.3 & 1.3 & 1.2 & 1.2 & 1.2 & 1.3 & 1.3 & $1.4^{*}$ & $1.3^{*}$ & 1.3 & 1.3 & 1.4 \\
\hline head depth & - & 2.6 & 2.3 & 2.3 & 2.3 & 2.5 & 2.3 & 2.1 & 2.3 & 2.3 & 2.2 & 2.4 & $2.7^{\circ}$ & 2.3 & 2.6 & 2.7 \\
\hline body depth at dorsal & - & 2.2 & 1.8 & 1.9 & 1.9 & 2.1 & 1.9 & 1.9 & 2.1 & 2.0 & 2.0 & 2.0 & 2.4 & 1.9 & 2.4 & 2.4 \\
\hline body width at dorsal & 1.6 & 1.7 & 1.4 & 1.4 & 1.5 & 1.5 & 1.4 & 1.4 & 1.5 & 1.5 & 1.5 & 1.5 & 1.7 & 1.5 & 1.5 & 1.6 \\
\hline body width at anal & 1.9 & 1.9 & 1.8 & 1.6 & 1.8 & 1.8 & 1.8 & 1.7 & 1.8 & 1.8 & 1.8 & 1.6 & 1.9 & 1.6 & 1.9 & 1.9 \\
\hline depth caudal peduncle & 12.5 & 11.9 & 7.9 & 9.1 & 7.7 & 11.0 & 10.2 & 10.0 & 10.7 & 10.3 & 11.6 & 11.8 & $14.8^{*}$ & 9.8 & 10.9 & 12.8 \\
\hline width caudal peduncle & - & 6.1 & 5.2 & 5.8 & 5.7 & 5.4 & 5.8 & 5.2 & 5.9 & 5.7 & 5.8 & $5.9^{*}$ & $6.0^{*}$ & 6.1 & 6.1 & 6.0 \\
\hline rictal barbel & - & 2.4 & 1.9 & 2.3 & \pm 2.1 & 1.8 & 2.0 & 2.1 & 2.3 & 2.3 & 2.4 & $1.7 *$ & $2.4^{\circ}$ & - & 2.0 & 2.2 \\
\hline lateral scutes & $33 / 32$ & $32 / 32$ & $32 / 32$ & $32 / 32$ & $32 / 32$ & $32 / 32$ & $32 / 32$ & $32 / 32$ & $32 / 32$ & $32 / 32$ & $32 / 32$ & $34 / 34^{*}$ & $33 / 33^{*}$ & $33 / 33$ & $33 / 33$ & $33 / 33$ \\
\hline coalescing scutes & $22 / 21$ & $20 / 21$ & $20 / 21$ & $22 / 21$ & $21 / 20$ & $21 / 20$ & $20 / 20$ & $20 / 20$ & $22 / 21$ & $20 / 20$ & $20 / 20$ & $19 / 19^{\circ}$ & $18 / 19$ & $18 / 18$ & $22 / 22$ & $20 / 20$ \\
\hline thoracic scutes & $7 / 7$ & $6 / 6$ & $6 / 8$ & $8 / 5$ & $7 / 7$ & $6 / 6$ & $6 / 7$ & $6 / 8$ & $6 / 6$ & $6 / 6$ & $7 / 6$ & $6 / 6^{\circ}$ & $6 / 6^{\circ}$ & $6 / 6$ & $6 / 6$ & $6 / 6$ \\
\hline teeth upper jaws & $5 / 5$ & $5 / 3$ & $14 / 14$ & $9 / 11$ & $>5 />5$ & $6 / 7$ & $7 / 9$ & $9 / 10$ & $9 / 9$ & $6 / 6$ & $6 / 7$ & $38 / ? 8$ & $? 4 /-$ & $-1>2$ & $11 / 12$ & $10 / 10$ \\
\hline teeth lower jaws & $?$ & $8 / 8$ & $17 / 16$ & $11 / 11$ & - & $12 / 14$ & $11 / 10$ & $11 / 11$ & $10 / 11$ & $11 /-$ & $-/ 10$ & $6 / 9$ & $9 / 5$ & $9 / 6$ & $13 / 14$ & $10 / 12$ \\
\hline
\end{tabular}

interorbital than found in any other Loricariichtbys species, including L. ucayalensis and L. maculatus. To avoid a lengthy technical discussion, the relation of standard length and interorbital width as a ratio of head length is shown in fig. 9. As can be seen in this figure, the interorbital of smaller specimens is usually narrower than that of adults. Amongst all Loricariichthys specimens we have measured thus far, only four specimens have a rather broad interorbital, approaching that of $L$. platymetopon: one is the lectotype of L. ucayalensis, another is the holotype of Parabemiodon typus, whereas the last two are syntypes of Loricariichthys anus (Valenciennes, in Cuvier \& Valenciennes, 1840).

Several specimens, including primary type- material of 14 of the 17 species described in the genus Loricariichthys (cf. Isbrücker \& Nijssen, 1976b: 110), were available for comparison with L. platymetopon. This material will form the basis for a revision of the genus and in this revision this material will be fully indicated.

Vaillant (1880: 157 \& 158) refers Loricaria maculata (non Bloch, 1794) sensu Valenciennes (in d'Orbigny, 1847, pl. VI fig. 3 “...et les deux figures au trait non numérotées.") to the synonymy of his Loricaria valenciennesii. Obviously, Vaillant did not re-examine Valenciennes's specimen, which undoubtedly is a Loricariichtbys platymetopon, whereas Loricaria valenciennesii is a junior synonym of Rineloricaria teffeana (Steindachner, 1878). 


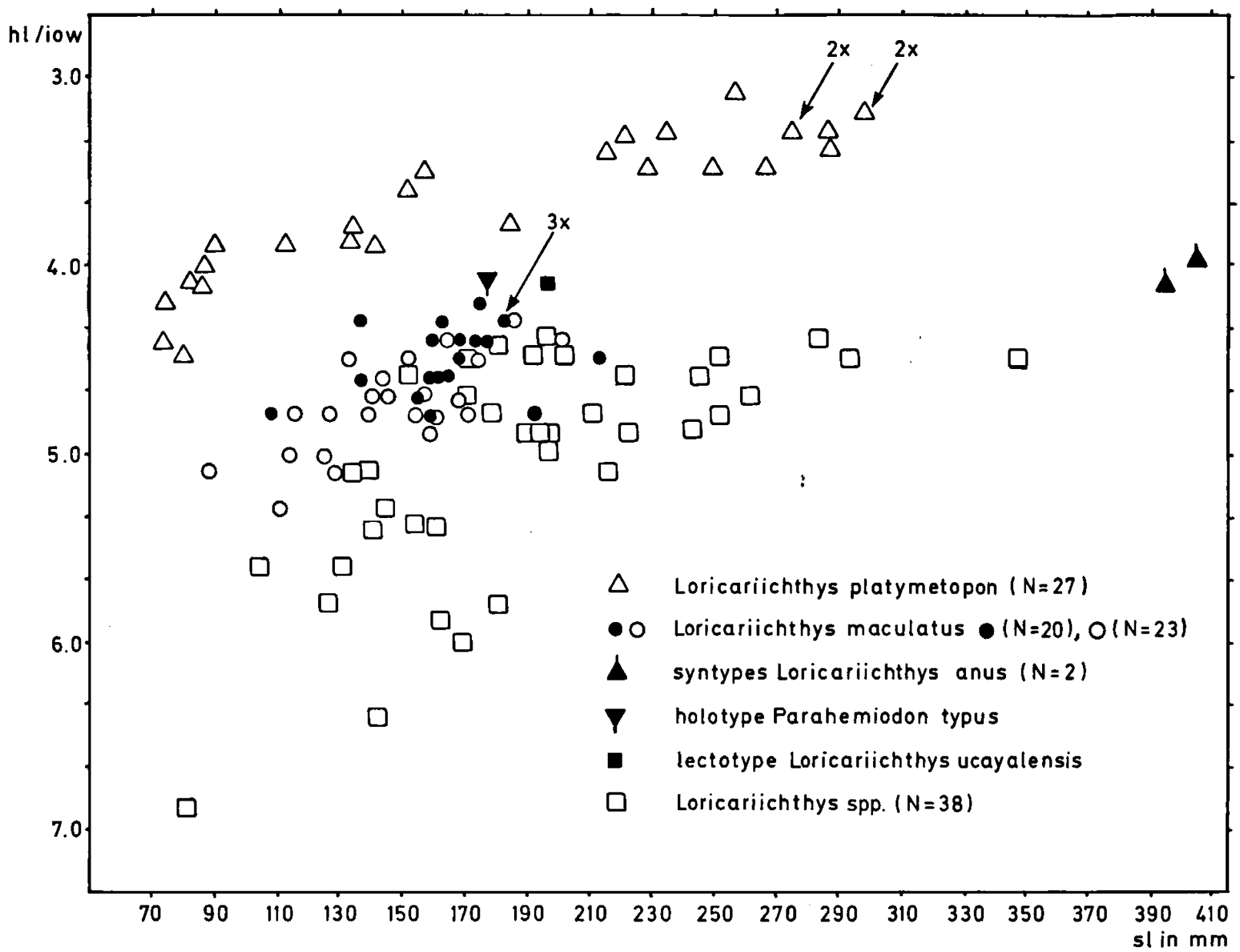

Fig. 9. Relation of the ratio head length/interorbital width and standard length in 112 specimens of several species of Loricariichthys, showing the wide interorbital area of Loricariichtbys platymetopon $\mathrm{n}$. $\mathrm{sp}$. The values shown as open circular symbols are taken from Boeseman (1976: 162, table 2), the other values are based on own observations.

\section{REFERENCES}

Bleeker, P., 1858. Ichthyologiae archipelagi Indici prodromus I. Siluri: v-viii, 1-370 (Lange \& Co., Batavia).

- 1862. Atlas ichthyologique des Indes Orientales Néêrlandaises, publié sous les auspices du Gouvernement colonial néêrlandais: Siluroïdes, Chacoïdes et Hétérobranchoïdes, 2: 1-112, pls. IL-CI (Fr. Muller, Amsterdam).

- 1864. Description des espèces de Silures de Suriname, conservées aux musées de Leide et d'Amsterdam. $\mathrm{Na}$ tuurk. Verh. holland. Maatsch. Wet. Haarlem, (2) 20: 1-104, pls. I-XVI.

BıOCH, M. E., 1794. Allgemeine Naturgeschichte der ausländischen Fische, 8: 1-174, pls. CCCLXI-CCCLXXXXVI (= Allgemeine Naturgeschichte der Fische, 11) (J. Morino \& Comp., Berlin).

Boeseman, M., 1976. A short review of the Surinam Loricariinae; with additional information on Surinam Harttiinae, including the description of a new species (Loricariidae, Siluriformes). Zoöl. Meded. Leiden, 50 (11): 153-177, pls. I-VIII.

BOULENGER, G. A., 1896. On a collection of fishes from the Rio Paraguay. Trans. zool. Soc. Lond., 14, II (1): 25-39, pls. III-VIII.

EIgenmanN, C. H., 1909. Reports on the Expedition to British Guiana of the Indiana University and the Carnegie Museum, 1908. Report No. 1. Some new genera and species of fishes from British Guiana. Ann. Carneg. Mus., 6 (1): 4-54.

- 1910. Catalogue and bibliography of the fresh water fishes of the Americas south of the tropic of Cancer. Catalogue of the fresh-water fishes of tropical and South temperate America. Rep. Princeton Univ. Exped. Patagonia, 1896-1899, 3 (Zool. 4): 375-511.

- 1912 . The freshwater fishes of British Guiana, including a study of the ecological grouping of species and the relation of the fauna of the plateau to that of the lowlands. Mem. Carneg. Mus., 5: i-xix, 1-578, pls. I-CIII.

Eigenmann, C. H. \& W. R. Allen, 1942. Fishes of western South America: 1.494, pls. I-XXII, 1 map (Univ. Kentucky, Lexington).

Eigenmann, C. H. \& R. S. Eigenmann, 1889. Preliminary notes on South American Nematognathi, II. Proc. Calif. Acad. Sci., (2) 2: 28-56. 
- \& -1890 . A revision of the South American Nematognathi, or cat-fishes. Occ. Pap. Calif. Acad. Sci., 1: 1.508, 1 map [also reprinted in facsimile in 1971 by Johnson Reprint Corporation, New York/London].

_ \& - 1891. A catalogue of the fresh-water fishes of South America. Proc. U.S. natn. Mus., 14: 1-81.

FowLER, H. W., 1940. A collection of fishes obtained by Mr. William C. Morrow in the Ucayali River Basin, Peru. Proc. Acad. nat. Sci. Philad., 91: 219-289.

—, 1945. Los peces del Peru. Catálogo sistemático de los peces que habitan en aguas peruanas: 1-298 (Mus. Hist. nat. "Javier Prado", Univ. San Marcos, Lima).

- 1954 . Os peixes de água doce do Brasil, 4. Arq. zool. S. Paulo, 9: i-ix, 1-400.

Gosline, W. A., 1945. Catálogo dos nematognathos de água-doce da América do Sul e Central. Bolm. Mus. nac. Rio de J. (n.s., Zool.), 33: 1-138.

GüNTHer, A. [C. L. G.], 1864. Catalogue of the Physostomi, containing the families Siluridae, Characinidae, Haplochitonidae, Sternoptychidae, Scopelidae, Stomiatidae, in the collection of the British Museum. In: Catalogue of the fishes in the British Museum, London, 5: i-xxii, 1.455 (Trustees British Museum, London).

HANCOCK, J., 1828. Notes on some species of fishes and reptiles, from Demerara, presented to the Zoological Society by John Hancock, Esq., Corr. Memb. Zool. Soc. In a letter addressed to the Secretary of the Society. Zool. J. Lond., 4: 240-247.

ISBRÜCKER, I. J. H., 1971. A redescription of the South American catfish Loricariichthys maculatus (Bloch, 1794), with designation of the lectotype and restriction of its type locality (Pisces, Siluriformes, Loricariidae). Bijdr. Dierk., 41 (1): 10-18.

ISBRÜCKER, I. J. H. \& H. NIJSSEN, 1976a. Rineloricaria heteroptera, a new species of mailed catfish from Rio Amazonas near Manaus, Brazil (Pisces, Siluriformes, Loricariidae). Zool. Anz., 196 (1-2): 109-124.

\& $1976 \mathrm{~b}$. The South American mailed catfishes of the genus Pseudoloricaria Bleeker, 1862 (Pisces, Siluriformes, Loricariidae). Beaufortia, 25 (325): 107-129.

_ $\&-1978$. Two new species and a new genus of neotropical mailed catfishes of the subfamily Loricariinae Swainson, 1838 (Pisces, Siluriformes, Loricariidae). Beaufortia, 27 (339): 177-206.

KNER, R., 1854. Die Panzerwelse des k. k. Hof-NaturalienCabinetes zu Wien. I. Abtheilung: Loricarinae. Denkschr. Akad. Wiss. Wien, mathem.-naturwiss. Cl., 6: 65-98, pls. I-VIII.

LüLING, K. H., 1975. Wissenschaftliche Ergebnisse der Argentinien-Südbrasilien-Expedition Dr. K. H. Lüling 1970/1971 [etc.]: Zool. Anz. Jena, 195 (3-4): 171-18.5.

Machado Allison, A. \& H. López Rojas, 1975. Etapas del desarrollo de Loricariichthys typus (Bleeker) 1864 (Osteichthyes, Siluriformes, Loricariidae). Acta biol. venez., 9 (1): 93-119.

Mago Leccia, F., 1970. Lista de los peces de Venezuela, incluyendo un estudio preliminar sobre la ictiogeografía del pais: 1-275, 31 unnumbered photographs (Oficina nacional de Pesca, Caracas).

Miranda Riberro, A. De, 1918. Lista dos peixes brasileiros do Museu Paulista, I. Revta. Mus. paul., 10: 707-736.
Müller, J. \& F. H. Troschel, 1848. Fische: 618-644. In: R. SchomburgK, ed. Reisen in Britisch-Guiana in den Jahren 1840-1844, [etc.], 3: i-viii, 533-1260 (J. J. Weber, Leipzig).

ØRVIG, T., 1977. A survey of odontodes ('dermal teeth') from developmental, structural, functional, and phyletic points of view. In: Mahala ANDREws, S., R. S. Mrles \& A. D. WALKer eds., Problems in vertebrate evolution. Linn. Soc. Symp. Ser., 4: 53-75, pls. I-III (published for the Linnean Society of London by Academic Press, London)

Pellegrin, J., 1899. Note sur les poissons recueillis par M. F. Geay dans l'Apuré et ses affluents. Bull. Mus. Hist. nat. Paris, 5 (4): 156-159.

REGAN, C. T., 1904. A monograph of the fishes of the family Loricariidae. Trans. zool. Soc. Lond., 17 (3): 191-350, pls. IX-XXI.

- 1913. Fishes from the River Ucayali, Peru, collected by Mr. Mounsey. Ann. Mag. nat. Hist., (8) 12: 281-283.

Ringuelet, R. A., R. H. ARÁmburu \& A. Alonso de ARÁmburu, 1967. Los peces argentinos de agua dulce: 1-602, pls. I-X (Provincia Buenos Aires, Gobernación, Comisión de Investigación Científica, La Plata).

SCHINDLER, O., 1959. Loricariichthys melini nov. sp. Ark. Zool., (2) 12 (26): 387-389.

SCHulTZ, L. P., 1944. The catfishes of Venezuela, with descriptions of thirty-eight new forms. Proc. U.S. natn. Mus., 94: 173-338, pls. I-XIV.

— 1949. A further contribution to the ichthyology of Venezuela. Proc. U.S. natn. Mus., 99: 1-211, pls. I-III.

SteINDACHNER, F., 1878. Zur Fisch-Fauna des MagdalenenStromes. Denkschr. Akad. Wiss. Wien, mathem.-naturwiss. $\mathrm{Cl}$., 39: 19-78, pls. I-XV.

—, 1915. Beiträge zur Kenntnis der Flussfische Südamerikas, V. Denkschr. Akad. Wiss. Wien, mathem.naturwiss. Kl., 93: 15-106, pls. I-XIII.

StIGCHEL, J. W. B. VAN DER, 1946. South American Nematognathi: 1-204, 3 tabs. (Thesis, State University of Leiden) (E. J. Brill, Leiden).

- 1947. The South American Nematognathi of the museums at Leiden and Amsterdam. Zoöl. Meded. Leiden, 27 (1.2): 1.204, 3 tabs.

Tovar Serpa, A., 1967. Peces del oriente peruano. Algunas especies de Loricariidae con referencia especial de la "carachama" Pteyrgoplichthys multiradiatus (Hancock), ecología y utilidad. Biota, Lima, 6 (50): 201-255.

VaIllant, L., 1880. Synopsis des espèces de Siluridae tecueillis par M. le Dr. Jobert, à Calderon (HauteAmazone). Bull. Soc, philomath. Paris, (7) 4: 150-159.

VAlenciennes, A., 1840. In: G. [L. C. F. D.] Cuvier \& A. Valenciennes, Histoire naturelle des poissons, 15 (Paris edition): i-xxxi, 1-540 (Strasbourg edition: i-xxiv, $1-397$, ii), pls. CCCCXXI-CCCCLV (Ch. Pitois, Paris \& V. Levrault, Strasbourg).

- 1847. Poissons. In: A. D'OrBIGNY ed., Voyage dans l'Amérique méridionale (Le Brésil, la République orientale de l'Uruguay, la République Argentine, la Patagonie, la République du Chili, la République de Bolivia, la République du Pérou), 5 (2): 1-11, pls. I-XVI (P. Bertrand, Paris \& V. Levrault, Strasbourg). 